\title{
EL RAZONAMIENTO CAUSAL SECUENCIAL EN LOS EQUILIBRIOS ÁCIDO-BASE MÚLTIPLES: PROPUESTAS DIDÁCTICAS EN EL ÁMBITO UNIVERSITARIO
}

\author{
JIMÉNEZ LISO, M. RUT, ${ }^{1}$ DE MANUEL TORRES, ESTEBAN ${ }^{2}$ y SALINAS LÓPEZ, FRANCISCO ${ }^{3}$ \\ ${ }^{1}$ Departamento de Didáctica de la Matemática y de las Ciencias Experimentales. Universidad de Almería \\ ${ }^{2}$ Departamento de Didáctica de las Ciencias Experimentales. Universidad de Granada \\ ${ }^{3}$ Departamento de Química Analítica. Universidad de Extremadura. Badajoz
}

\begin{abstract}
Resumen. La principal dificultad que encuentran los estudiantes al acercarse a los equilibrios ácido-base múltiples es que precisan controlar varios equilibrios de forma simultánea. Una anticipación en la presentación a los estudiantes de educación secundaria de estos equilibrios induce a la utilización del razonamiento causal secuencial, por el cual se consideran los procesos no como equilibrios sino como compartimentos estanco (sin simultaneidad) y cada proceso como consecuencia del anterior y como causa del siguiente. En el presente artículo, por un lado, diagnosticamos las concepciones alternativas relacionadas con los equilibrios ácido-base múltiples (estudiantes universitarios, licenciados-opositores a la educación secundaria y libros universitarios; y por el otro, se centra en una propuesta de mejora del aprendizaje utilizando para ello la resolución de problemas y el ordenador como recurso auxiliar en esta resolución. Previamente analizamos los enunciados de los problemas que se plantean en algunos libros universitarios como punto de partida a nuestra propuesta didáctica, la cual se centra en que los estudiantes expliciten todos los equilibrios presentes en los procesos ácido-base múltiples como parte de una heurística para la resolución de los problemas y utilicen el ordenador para salvar las dificultades matemáticas primando el análisis del proceso químico.
\end{abstract}

Palabras clave. Ácido-base, razonamiento secuencial, concepciones alternativas, universitarios, propuestas didácticas.

Summary. The need to control several equilibria simultaneously is the main difficulty met by students when they approach multiple acid-base equilibria. Presenting beforehand these equilibria to secondary school students encourages the use of causal sequential reasoning that takes processes not as equilibria but isolated, without simultaneity, each process being the cause of the former and causing the following. In this article, on the one hand we diagnose the alternative conceptions related to multiple acid-base equilibria. On the other hand we propose to improve learning by using problem solving and the computer as an auxiliary resource. We previously analyse the wording of the problems presented in some university textbooks as starting point to our didactic proposal, which asks the students to explain all the equilibria in multiple acid-base processes as part of a heuristic for problem solving and to use the computer to solve mathematical difficulties, giving priority to the analysis of the chemical process.

Keywrods. Acid-base, sequential reasoning, alternative conceptions, university students, teaching strategies.

\section{INTRODUCCIÓN}

El «razonamiento causal secuencial» es descrito por Viennot (1979) y por Driver y otros (1989) en relación con las concepciones de los alumnos sobre los circuitos eléctricos sencillos. Estos autores destacan un tipo de respuestas (antes y después) que constituye un modelo de circuito en el que cada elemento influye sobre lo que está 
detrás de él, pero no sobre lo que está antes. Este modelo utilizado habitualmente por los alumnos es denominado como modelo dependiente del tiempo o como modelo secuencial (para denotar la influencia de factores, tanto espaciales como temporales).

Para los estudiantes (y para la gente en general) una causa produce una cadena de efectos con una secuencia temporal; por ejemplo, los estudiantes tienden a pensar que una fuerza (causa) produce un movimiento (efecto) y les resulta difícil apreciar las interacciones recíprocas (tercera ley de Newton) porque supone abandonar el modo de pensamiento secuencial en su dirección «preferida» (Driver et al., 1989). Estas preferencias por una «dirección» parecen ser el origen de que le otorguen el carácter de irreversible a los equilibrios químicos. A este respecto, Andersson (1990) destaca que los alumnos utilizan razonamientos de «causalismos simples» al interpretar los procesos de cambio químico.

Otra de las posibles causas de la utilización del pensamiento lineal es señalada por Sanmartí (1996), quien, al insistir en que en las clases de ciencias se incide poco en el aprendizaje del lenguaje en general y de los conectores en particular, señala que en las descripciones de clase se abusa del «entonces... y entonces... y entonces...» que refuerzan el pensamiento lineal, dificultando que los estudiantes accedan al pensamiento multicausal y complejo.

Las investigaciones sobre las estrategias del procesamiento de la información, concretamente los estudios de Pask (1976, 1988) y Pask y Scott $(1972,1973)$ destacan dos estrategias de aprendizaje fundamentales: la holística y la secuencial. Entre las características descritas para la estrategia secuencial del procesamiento de la información destacan el procesamiento lineal y secuencial centrándose en un solo tópico, con aprendizaje local, reproductivo, desarrollando hipótesis simples y produciendo una comprensión fragmentada de manera que las relaciones entre los ejemplos y las aplicaciones a la vida real se secuencian y ralentizan, mientras que el aprendizaje holístico permite considerar varios tópicos al mismo tiempo, ya que el aprendizaje es global, produciéndose generalizaciones, desarrollando hipótesis complejas, integrando la teoría y la vida real, etc. (Ford, 2000).

En el presente artículo nos centraremos en la utilización del razonamiento causal secuencial en los procesos de equilibrios ácido-base múltiples. En un trabajo anterior (Jiménez-Liso et al., 2001), pusimos de manifiesto que, al estudiar los procesos ácido-base en los que intervenían ácidos fuertes con bases débiles o viceversa, es frecuente justificar que el $\mathrm{pH}$ de la disolución final es diferente de 7 porque primero se forma la sal y, posteriormente, ésta se hidroliza. Este modo de justificar el fenómeno ignora que desde un principio se dan todos los equilibrios posibles de todas las especies que intervienen en el proceso, inmediatamente y simultáneamente. No tener esto en cuenta significa caer en un razonamiento causal secuencial, que consiste en suponer que los equilibrios ocurren sucesivamente y cada etapa es efecto de una causa y, a su vez, causa de la siguiente etapa:
Reacción ácido-base $\rightarrow$ neutralización $\rightarrow$ hidrólisis $\rightarrow \mathrm{pH} \neq 7$.

Antes de describir el estudio exploratorio sobre la utilización, por los estudiantes universitarios, de este tipo de razonamiento en los equilibrios ácido-base múltiples, comenzaremos con una breve revisión de los trabajos previos que hay sobre este tema.

\section{ANTECEDENTES}

Las concepciones alternativas sobre los procesos ácidobase han sido indagadas por numerosos autores. Algunos se centran en las dificultades de los estudiantes de educación secundaria para clasificar las sustancias como ácidas, como neutras o como básicas (Cros, 1986, 1988; Zoller, 1990; Ross y Munby, 1991; Nakhleh y Krajcik, 1994; Vidyapati y Seethramappa, 1995; Toplis, 1998). La utilización de términos como neutralización (Schmidt, 1991) o hidrólisis (Jiménez-Liso, 2000) con varios significados puede ser el origen de algunas concepciones alternativas como la identificación de neutralización con cualquier proceso ácido-base y la extrapolación de que, cuando un ácido reacciona con una base, el producto final siempre es neutro. Algunos autores han detectado que la mayoría de los estudiantes, incluso los universitarios, no beberían disoluciones ácidas (Cros, 1986, 1988; Jiménez-Liso et al., 2000) porque consideran que son fuertes, poderosas y dañinas (Toplis, 1998) para el organismo y destacan que los valores bajos del $\mathrm{pH}$ se deben a la presencia de ácidos fuertes o a la elevada concentración de los ácidos presentes (Fortman, 1994; Jiménez-Liso, 2000).

Los indicadores ácido-base también constituyen un contenido clave en la enseñanza de los procesos ácido-base. Baber (1996), Cobb (1998) y VanCleave (1998) describen el uso de un indicador fabricado a partir de un producto casero como es el jugo de la lombarda y las ventajas de la aplicación de esta actividad para alumnos de educación secundaria. Kanda y otros (1995) proponen una actividad para preparar bolas camaleónicas utilizando extractos de plantas y ofrecen una tabla de los colores que muestran diferentes plantas a distintos valores del $\mathrm{pH}$. Uzelmeier y Breyer (1998) muestran a los alumnos universitarios las diferencias entre los indicadores ácido-base que cambian de color si se le añade ácidos o bases, frente a los colorantes alimenticios que no cambian de color por la adición de esas sustancias. De esta forma llaman la atención sobre el peligro de identificar algunos colores con sustancias ácidas o básicas sin tener en cuenta los cambios químicos en el caso de los indicadores ácido-base.

Por otro lado, el razonamiento causal secuencial descrito en la introducción del presente artículo puede ser el origen de algunas concepciones alternativas como la que señalan De la Guardia y otros (1985), quienes encontraron que los alumnos de los niveles universitario y medio asignaban el valor 7 al pH del punto de equivalencia en cualquier valoración ácido-base; incluso el $30 \%$ de los estudiantes de $5^{\circ}$ curso de la licenciatura en químicas, especialidad en química analítica, daba eso por sentado. Estos autores rastrearon en libros de educación secundaria y bachillerato 
encontrando en ellos formulaciones equívocas o ejemplos simplistas que pudieran, al generalizarse por los alumnos, dar lugar a las concepciones alternativas observadas. Este rastreo les reveló la presencia de abundantes ejemplos de valoraciones de un ácido fuerte con una base fuerte y, tal vez, esto podría inducir a los alumnos a generalizar que, en el punto de equivalencia, el valor del $\mathrm{pH}$ siempre es 7.

A continuación, expondremos los objetivos de nuestro trabajo que, en una primera fase, se centran en el diagnóstico de concepciones alternativas relacionadas con los procesos ácido-base múltiples y, en una segunda etapa, se plantea una propuesta de mejora de la resolución de problemas relacionados con el tema.

\section{OBJETIVOS Y PLANTEAMIENTO DEL TRA- BAJO}

En este artículo queremos conseguir fundamentalmente dos objetivos: uno relacionado con la indagación de las con- cepciones alternativas que manejan los estudiantes universitarios y los licenciados, sobre los equilibrios ácido-base, y otro relacionado con una propuesta de modificación de los problemas que se plantean en estos niveles para facilitar el aprendizaje de los equilibrios ácido-base múltiples y evitar la inducción de las concepciones alternativas diagnosticadas.

Por todo ello, expondremos los resultados de un estudio exploratorio sobre las concepciones de un grupo numeroso $(\mathrm{N}=450)$ de alumnos universitarios de la licenciatura de químicas, de una muestra de licenciados que aspiran a acceder al cuerpo de profesores de educación secundaria (opositores) y los contenidos que aparecen en los libros de los niveles universitario y medio.

Los resultados ponen de manifiesto que una de las causas del razonamiento causal secuencial, antes descrito, puede ser el estudio prematuro de los equilibrios múltiples en los niveles medios. Al «adelantar acontecimientos», el docente tiene que recurrir a «estrategias didácticas» (equilibrios por etapas) que ocasionan el razonamiento causal

\section{Equilibrios ácido-base múltiples}

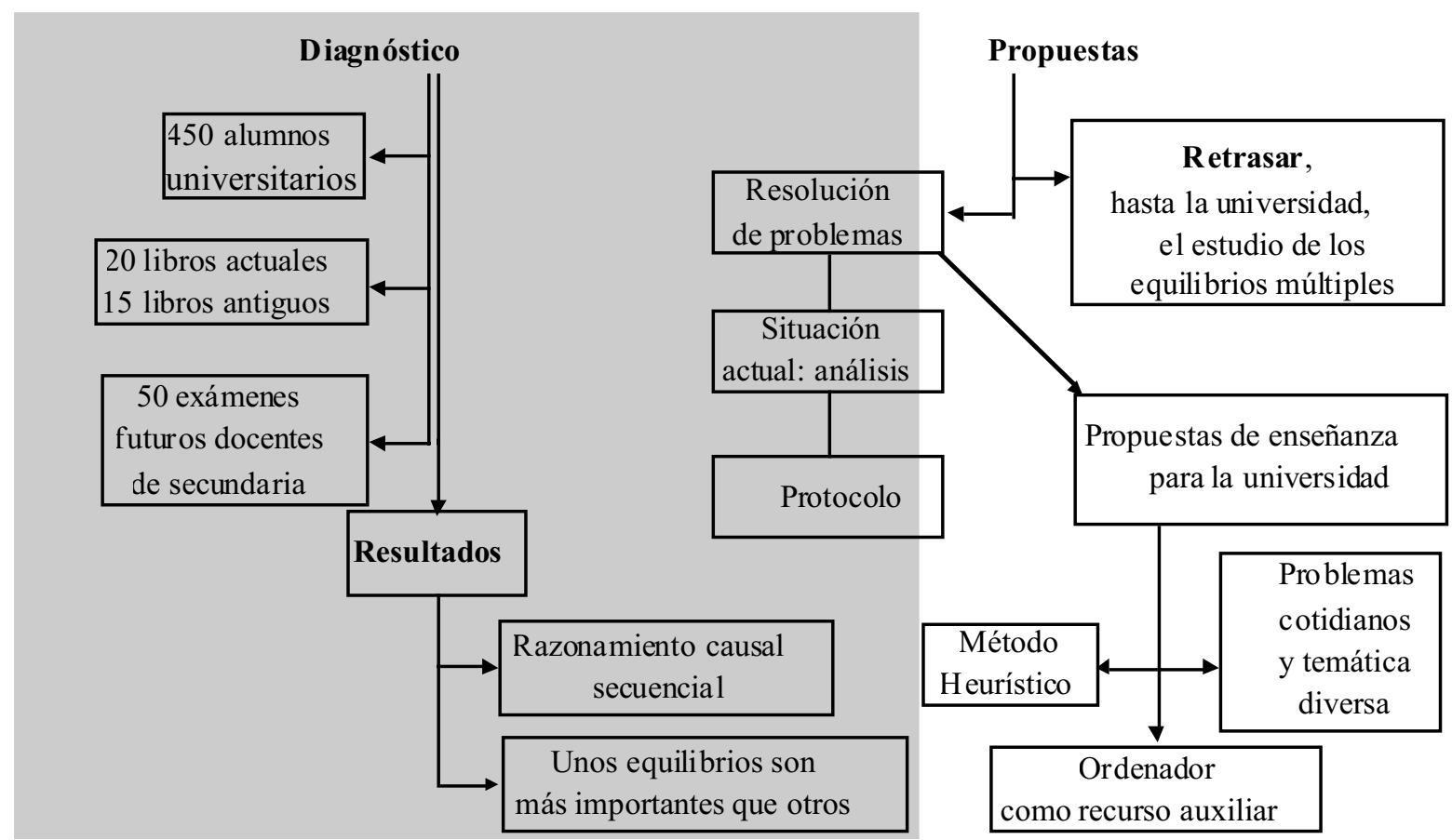


secuencial, para que los alumnos interpreten de una forma más sencilla los equilibrios múltiples que, por su dificultad, deberían ser estudiados en los niveles universitarios.

Otra de las posibles causas del pensamiento lineal en los equilibrios múltiples puede ser la deficiente utilización de la resolución de problemas sobre este tema. Esta actividad puede ofrecer una buena ocasión para que los alumnos utilicen y controlen las variables que intervienen en los procesos ácido-base de varios equilibrios. Con el objetivo de mejorar la enseñanza de los procesos ácido-base múltiples, indagaremos si es necesario modificar los problemas que se suelen plantear en los niveles universitarios. Para ello, presentaremos un análisis de los problemas propuestos y del proceso de resolución que desarrollan varios libros universitarios sobre los equilibrios ácido-base, con el fin de constatar si reducen el número de equilibrios presentes en un proceso ácido-base, induciendo la idea de que unos son más importantes que otros e, incluso, que algunos son innecesarios. Las aproximaciones que realizan en su desarrollo suponen un alejamiento de la realidad: al eliminar equilibrios químicos, porque no interesan para la resolución, se concede más importancia al proceso matemático (obtención de un resultado numérico) que a una interpretación química y, con el paso del tiempo, se termina por no considerar este aspecto.

Por último, expondremos una propuesta concreta de utilización de los problemas y de su resolución con el fin de mejorar el aprendizaje de los equilibrios múltiples de los estudiantes universitarios. De esta forma, mostraremos algunos problemas heurísticos (requieren una estrategia previamente planificada, según la clasificación de Perales, 2000) y otros creativos (aquéllos que pueden resolverse de diferentes formas sin estrategia predeterminada), con temática diversa, de forma que no centremos la atención exclusivamente sobre el cálculo del $\mathrm{pH}$ o de la concentración de iones $\mathrm{H}_{3} \mathrm{O}^{+}$, sino que insistamos por igual en todas las especies presentes en el proceso. Esta insistencia, unida a la de utilizar todos los equilibrios posibles, ayudará a una mayor comprensión del problema «químico» relegando a un segundo plano el «problema matemático». Como veremos, nuestra propuesta se centra en dar una mayor importancia a los conceptos químicos mediante la explicitación de todos los equilibrios presentes en el proceso, la deducción de las ecuaciones que permitan al alumno calcular las concentraciones de todas las especies y restar importancia al cálculo matemático, utilizando el ordenador como instrumento auxiliar (Jonassen, 1999) que permita resolver las ecuaciones. Por último, tras conseguir de esta forma los resultados numéricos, provocaremos una discusión o análisis cualitativo de todo el problema para volver a llamar la atención del alumno sobre el proceso químico y no limitarnos a obtener un resultado numérico.

En la figura 1 mostramos el planteamiento del artículo, destacando (en el recuadro punteado) la fase de diagnóstico relacionada con las concepciones sobre los equilibrios múltiples (alumnos, libros y profesores) así como la relativa a los problemas que se proponen en los libros y a su resolución. Las propuestas están fundamentadas en los resultados de esa fase (retraso de los equilibrios hasta los niveles universitarios y propuestas de mejora de los problemas que se planteen sobre equilibrios múltiples y su resolución).

\section{EL RAZONAMIENTO CAUSAL SECUENCIAL EN LOS EQUILIBRIOS MÚLTIPLES: FASE DE DIAGNOSTICO}

Como hemos indicado en la introducción, vamos a exponer la indagación de las concepciones alternativas que hemos abordado en nuestra investigación, señalando la metodología empleada (pruebas, protocolos de análisis de contenidos en textos escritos, etc.), las muestras diagnosticadas (alumnos universitarios, licenciados, libros, etc.), los resultados obtenidos y su análisis, la cual nos permitirá fundamentar nuestra propuesta de enseñanza de los contenidos implicados y facilitar su aprendizaje en los niveles universitarios.

\section{Concepciones de los estudiantes universitarios sobre los equilibrios ácido-base múltiples}

Para la indagación de las concepciones de los estudiantes universitarios sobre los contenidos relacionados con los procesos ácido-base, diseñamos una prueba semiabierta (Jiménez-Liso, 2000) de opciones múltiples en la que se solicitaba a los alumnos que justificaran su elección para comprobar la seguridad en sus respuestas y para que pudieran explicitar alguna idea que no estuviera recogida en las diferentes opciones. La prueba fue aplicada a una muestra de 450 estudiantes universitarios de los cinco cursos de la licenciatura de ciencias químicas en varias universidades españolas durante el curso 1997-98.

En dicha prueba incluimos varios ítems relacionados con las teorías ácido-base y sus límites de aplicación, la neutralización, la hidrólisis, la operación de conservación de la cantidad de sustancia en las valoraciones, etc. De la prueba, y para el propósito de este trabajo, destacaremos tres ítems: en uno de ellos se indagaba sobre la utilización de todos los equilibrios (incluido el del indicador) y en los otros dos ítems se buscaba constatar si los alumnos consideran la existencia de los equilibrios entre todas las especies químicas que intervienen en una valoración o en un proceso estequiométrico. Además, en estos dos ítems se indagaba si los estudiantes razonaban secuencialmente el proceso, es decir, si consideraban una secuencia de pasos para los equilibrios (reacción ácido-base $\rightarrow$ neutralización $\rightarrow$ hidrólisis $\rightarrow \mathrm{pH} \neq 7$ ). A continuación mostramos los dos ítems utilizados:

Eq-1. Si se van añadiendo $n$ moles de $\mathrm{NaOH}$ (en disolución acuosa) a $n$ moles de $\mathrm{CH}_{3} \mathrm{COOH}$ (también en disolución acuosa) sucede lo siguiente:

a) Se forman $n$ moles de $\mathrm{NaCH}_{3} \mathrm{COO}(\mathrm{ac})$ y posteriormente se produce una hidrólisis, el $\mathrm{pH}$ serả mayor que 7 .

b) Al no haber exceso de moles del ácido ni de la base, se obtiene una disolución cuyo $\mathrm{pH}$ es 7.

c) En la disolución se cumplirá: $\left[\mathrm{CH}_{3} \mathrm{COO}^{-}\right]=\left[\mathrm{Na}^{+}\right]$y $\left[\mathrm{H}_{3} \mathrm{O}^{+}\right]=$ $[\mathrm{OH}]$; el pH será 7 . 
d) En la disolución habrá un número de moles de $\mathrm{CH}_{3} \mathrm{COO}^{-}, \mathrm{H}_{3} \mathrm{O}^{+}$, $\mathrm{OH}^{-}$tal que el $\mathrm{pH}$ será mayor que 7 .

e) Otras respuestas.

Justifique la respuesta.

Eq-2. Cuando se añade, gota a gota, una disolución en agua de $\mathrm{H}_{2} \mathrm{SO}_{4}$ a una disolución acuosa de $\mathrm{NH}_{3}$, en el punto de equivalen-

a) El equilibrio entre las especies químicas, que se da desde el principio, origina un $\mathrm{pH}$ final menor que 7 .

b) El pH será menor que 7 porque el viraje del indicador se produce por un exceso de ácido.

c) El pH será igual a 7, si bien, posteriormente la hidrólisis del $\mathrm{NH}_{4}^{+}$hará descender el $\mathrm{pH}$.

d) El pH será igual a 7 porque se ha añadido un número de equivalentes de ácido igual al de los que se pusieron de la base.

e) Otras respuestas.

Justifique la respuesta.

En la tabla I mostramos los porcentajes de alumnos que eligen cada opción de estos ítems (Jiménez-Liso et al., 2000). Las opciones elegidas por cada alumno fueron sometidas a un tratamiento estadístico sencillo que se detalla ampliamente en Jiménez-Liso (2000). Las respuestas abiertas para las justificaciones han sido agrupadas por similitud, categorizadas y jerarquizadas por la frecuencia de alumnos que las utilizan con el objetivo de poder analizarlas y compararlas con los resultados cuantitativos.

Tabla I

Porcentajes de alumnos que eligen cada opción.

\begin{tabular}{|l|c|c|c|c|c|c|}
\hline $\begin{array}{l}\text { Opción } \\
\text { Ittem }\end{array}$ & a & b & c & d & Otras & En blanco \\
\hline Eq-1 & 44 & 8 & 13 & $22 *$ & 5 & 7 \\
\hline Eq-2 & $12 *$ & 17 & 20 & 25 & 6 & 20 \\
\hline
\end{tabular}

* Los porcentajes marcados con el asterisco corresponden a los alumnos que aciertan cada ítem.

Los resultados del ítem Eq-1 ponen de manifiesto que la mayoría de los estudiantes se decanta por el razonamiento causal secuencial descrito en la opción $a$. En el ítem Eq-2, las respuestas mayoritarias se reparten entre el proceso secuencial $(20 \%)$ y el que se centra en la estequiometría $(25 \%)$. En las dos opciones se plantea que en el punto de equivalencia, se produce la neutralización $(\mathrm{pH}=7)$; sin embargo, una opción se centra exclusivamente en el proceso estequiométrico (en este valor del $\mathrm{pH}$ ) y la otra opción manifiesta la continuación del proceso (hidrólisis posterior que hace variar el $\mathrm{pH}$ ).

Las justificaciones de los estudiantes, al responder a estos dos ítems, confirman lo indicado en el estudio cuantitativo de las opciones múltiples. Citamos, como ejemplo, algunas frases textuales representativas por la frecuencia con que se repiten: «Se produce una reacción de neutralización primero y, por posterior formación del acetato sódico, se hidroliza el $\mathrm{CH}_{3} \mathrm{COO}^{-}$liberando $\mathrm{OH}^{-}$al medio, siendo $\mathrm{pH}>7 . »$ (alumno de $5^{\circ}$ curso) «Al ser reacción de neutralización se produce $\mathrm{CH}_{2} \mathrm{COONa}$ y $\mathrm{H}_{2} \mathrm{O}$ y, como el acetato proviene de ácido débil, sufre hidrólisis.» $\left(5^{\circ}\right.$ curso $)$ «Se produce la valoración del $\mathrm{AcH} \rightarrow \mathrm{AcNa}$, y como el $\mathrm{AcH}$ es ácido débil $\rightarrow$ base conjugada fuerte $\rightarrow$ se hidroliza $\rightarrow$ $\mathrm{pH}>7 . »$ (3r. curso) y lo mismo para las justificaciones al ítem Eq-2: «Justo en el punto de equivalencia, el $\mathrm{pH}=7$, posteriormente al hidrolizarse $\mathrm{NH}_{4}^{+}+\mathrm{H}_{2} \mathrm{O} \rightarrow \mathrm{NH}_{4} \mathrm{OH}+$ $\mathrm{H}^{+} . »\left(4^{\circ}\right.$ curso $)$ «En un principio el, $\mathrm{pH}=7$ en el punto de equivalencia, pero al pasar un tiempo desciende por la hidrólisis del $\mathrm{NH}_{4}^{+}$(3r. curso).

Después de analizar todas las respuestas abiertas (JiménezLiso, 2000), creemos que una de las causas de este razonamiento tan arraigado y persistente puede estar en la utilización del término neutralización para cualquier proceso ácido-base, como así se pone de manifiesto en las siguientes justificaciones: «En el punto de equivalencia se habrá neutralizado todo $\mathrm{NH}_{3}$ y su $\mathrm{pH}=7 . »\left(5^{\circ}\right.$ curso) «Neutralizamos la reacción, porque añadimos un ácido a una base. $\mathrm{Eq}_{\text {ác }}=$ eq $_{\text {base }} .{ }^{\circ} 4^{\circ}$ curso $)$ «Porque el punto de equivalencia es justo cuando en toda la base se ha neutralizado el ácido.» ( $4^{\circ}$ curso $)$ «Se neutralizan.» $\left(2^{\circ}\right.$ curso $)$ «El ácido y la base se neutralizarían.» (1r. curso). Este aspecto ha sido ampliamente desarrollado en un trabajo previo (JiménezLiso y De Manuel, 2002).

Con respecto a las justificaciones de la opción correcta, en algunos casos se expresa el razonamiento secuencial descrito anteriormente. Resulta curioso que un pequeño porcentaje de alumnos rechace la opción que se ofrece en el ítem Eq-1 que hace referencia al equilibrio por etapas y elijan la opción correcta. Esto parece manifestar que consideran que los equilibrios transcurren de forma simultánea y no secuencial, aunque no expresen todos los equilibrios que tienen lugar sino los que dan $\mathrm{OH}^{-}$como producto de la reacción y, por tanto, como argumento de por qué la disolución final es básica. El resto de las justificaciones a la opción correcta hace referencia a las fuerzas relativas del ácido y de la base presentes en los distintos procesos.

El tercer ítem que hemos destacado en párrafos anteriores tiene como objetivo fundamental contrastar si los estudiantes ponen en juego todos los equilibrios que intervienen en un proceso múltiple (incluido el equilibrio del indicador). En él se ofrecían diversas opciones: desde la que no tiene en cuenta ni el intervalo de viraje ni el equilibrio del indicador hasta la opción correcta en la que se consideran ambos factores. El ítem «Ind» quedó redactado de la siguiente forma:

Ind. En una valoración de un ácido, mediante una base que se añade desde la bureta, se emplea un indicador que en su forma ácida tiene un color y en su forma básica tiene un color diferente. Durante la valoración, el pH va aumentando y sucede lo siguiente:

a) En todo el proceso existen las dos formas del indicador; pero dentro de un intervalo de $\mathrm{pH}$, con la vista, apreciamos un color que es mezcla de los dos. 
b) Al principio, todo el indicador se encuentra en su forma ácida y al llegar a un valor del $\mathrm{pH}$ cambia totalmente a la forma básica y vira.

c) Existe un intervalo de $\mathrm{pH}$ en el que están presentes las formas ácida y básica del indicador; fuera de este intervalo, sólo existe una de las dos formas.

d) En todo el proceso existen las dos formas del indicador, y para un determinado valor del $\mathrm{pH}$ se produce un cambio total del color

e) Otras respuestas.

Justifique la respuesta.

Los resultados de este ítem indican que sólo un $8 \%$ de los estudiantes universitarios diagnosticados eligió la opción correcta, mientras que la opción en la que no se tiene en cuenta ni el intervalo de viraje ni que las dos formas del indicador coexisten siempre en equilibrio es elegida por el $39 \%$ de ellos. Un $28 \%$ eligió la opción $c$ (con intervalo - sin equilibrio) y un $14 \%$ optó por la respuesta $d$ ( $\sin$ intervalo - con equilibrio). Las justificaciones de los estudiantes confirman estos resultados.

A modo de resumen señalaremos que las principales concepciones diagnosticadas relacionadas con los procesos ácido-base son la utilización del razonamiento causal secuencial en los procesos múltiples y la consideración de dichos equilibrios como compartimentos estanco que no se desarrollan simultáneamente; y la ausencia de algunos equilibrios, por ejemplo, el del indicador, lo que manifiesta que se concede mayor importancia a unos equilibrios que a otros.

\section{Análisis de los contenidos sobre equilibrios ácido-base múltiples en los libros actuales y antiguos (1868-1955) de los niveles universitario y medio}

En este apartado queremos dar a conocer el análisis de una muestra de libros universitarios y de educación secundaria actuales y otra de comienzos del siglo xx con el objetivo de indagar el tratamiento que conceden a los procesos ácido-base múltiples y observar su desarrollo a lo largo del siglo xx. La razón principal de explorar estos libros es examinar las posibles relaciones entre los conceptos que se exponen en los textos y las concepciones que manifiestan los alumnos que los utilizan.

El instrumento diseñado para el análisis de los textos es un protocolo en el que se analizan los mismos contenidos que los indagados en los alumnos universitarios (las definiciones de ácidos y bases, los límites de aplicación de las teorías, los conceptos de neutralización e hidrólisis, la relación entre el $\mathrm{pH}$ y la cantidad de sustancia, el equilibrio y el intervalo de viraje del indicador, varios aspectos relacionados con las valoraciones y con las disoluciones reguladoras, etc.). De este modo, se puede realizar un estudio comparativo y analizar su influencia sobre las concepciones alternativas de los alumnos.

Los libros actuales seleccionados (15 universitarios y 5 de educación secundaria) constituyen una muestra represen- tativa de los textos más utilizados por los alumnos de la licenciatura de química de algunas universidades españolas y de los más vendidos para el segundo curso de bachillerato (17-18 años), editados después de la reforma educativa española. Un criterio utilizado para su selección ha sido que presentaran de forma amplia los contenidos relacionados con los procesos ácido-base, por ello abundan textos de química general, de química inorgánica y de química analítica en la muestra de libros analizada.

El protocolo también ha sido aplicado a una muestra de 15 libros editados con fechas comprendidas entre 1868 y 1944. De éstos, 9 son textos universitarios de diferentes materias y 6 libros de los niveles superiores de secundaria. Las muestras de libros actuales y antiguos son diferentes: entre los libros antiguos hay uno de química física y otro de prácticas de laboratorio mientras que no hay ningún texto de esas características en la muestra de libros actuales. En ésta no aparece ningún libro que no contenga un capítulo dedicado a los contenidos sobre los procesos ácido-base. A pesar de ello procede la comparación porque la información sobre estos contenidos que recibe un alumno universitario actual proviene de los libros de química general, inorgánica y analítica, mientras que un alumno de comienzos de siglo recibía la información sobre los procesos ácido-base de una gama más amplia de textos y de asignaturas.

El protocolo, las muestras de libros analizados y los resultados referentes a todos los contenidos analizados han sido desarrollados en trabajos previos (Jiménez-Liso, 2000; Jiménez-Liso et al., 2001). A continuación presentamos los contenidos relacionados con los equilibrios ácido-base múltiples. En primer lugar destacaremos que sólo en dos libros universitarios actuales se incluyen todos los equilibrios que intervienen en una valoración (o en una reacción estequiométrica) y en el $60 \%$ de los textos se indica que primero se forma la sal y, posteriormente, se produce la hidrólisis (razonamiento causal secuencial). Todos los libros de educación secundaria actuales analizados y el 19\% de los libros históricos (todos los que explican el proceso que transcurre en una valoración) muestran el citado razonamiento causal secuencial.

En más de la mitad de los textos universitarios actuales (67\%) no se expresa la idea de que las formas ácida y básica del indicador coexisten siempre en equilibrio. En dos de ellos aparece la siguiente idea: «[...] si se conoce el pH al que cambia de una forma a otra $[. .$.$] se puede determinar$ si una disolución tiene un $\mathrm{pH}$ mayor o menor que ese valor». Tampoco se expresa con claridad que existe un intervalo de viraje del indicador en el que el color que se aprecia es mezcla de los dos, sino que se suelen utilizar algunos términos como cambio «súbito [...] brusco [...] dramático [...] el indicador cambia de color a una cierta concentración del ácido, es decir, a un valor del $\mathrm{pH}$. El cambio no es bastante preciso, tiene lugar en un margen amplio».

Hay que destacar que los aspectos relacionados con los indicadores aparecen de forma correcta en el $80 \%$ de los libros de $2^{\circ}$ de bachillerato; sin embargo, no se explicita en ninguno de ellos que, en el intervalo de viraje, el color que se aprecia es una mezcla de los dos colores. 
Los libros antiguos muestran resultados similares, aunque con la diferencia de que más de la mitad de ellos (57\%) no menciona los indicadores. En el resto se pone de relieve la ausencia del equilibrio y el intervalo de viraje, como describe uno de ellos: «Los indicadores están constituidos por sustancias que reaccionan con la solución valorada, solamente cuando ha reaccionado toda la sustancia que se analiza o trata de determinar. Así: la fenolftaleina de la acidimetría reacciona con los álcalis, pero solamente cuando no hay ácido en presencia pues entonces éste lo hace con el álcali y la fenolftaleína queda inalterada; pero en el momento en que el ácido desaparece, la fenolftaleína tiene color rojo, que caracteriza, por consiguiente, la terminación de la reacción estudiada.»

Como hemos expuesto en un trabajo previo (Jiménez-Liso et al., 2001), todos los conceptos que se indagan en el protocolo aparecen en la mayoría de los textos de educación secundaria y se tratan de forma muy similar a como se presentan en los libros del nivel superior. El estudio de los procesos ácido-base se concentra en pocos cursos, entre $2^{\circ}$ de bachillerato y los primeros cursos de universidad fundamentalmente, lo que puede ocasionar dos inconvenientes: en primer lugar, los resultados señalan que se están repitiendo en varios cursos los mismos contenidos, lo que es una pérdida de tiempo si esos conceptos se tratan de igual forma; y, en segundo lugar, se tiende a adelantar el estudio de algunos conceptos que, por su dificultad, deberían ser incluidos en cursos superiores. Por ejemplo, todos los libros de $2^{\circ}$ de bachillerato analizados incorporan al capítulo de «reacciones de transferencia de protones» las valoraciones de ácidos débiles con bases fuertes o viceversa (equilibrios múltiples). Teniendo en cuenta que los equilibrios químicos no se estudian hasta el capítulo inmediatamente anterior al de ácidos y bases, se exige a los alumnos que acaban de conocer la existencia de los equilibrios que controlen todos los presentes en este complejo proceso.

Además, y lo que es bastante perjudicial, para que el alumno no tenga que realizar el esfuerzo de controlar todas esas variables, se propone una «estrategia didáctica» que consiste en considerar los equilibrios como reacciones sucesivas (primero se forma la sal y luego ésta se hidroliza). Esta estrategia didáctica provoca que los alumnos de educación secundaria utilicen un razonamiento causal secuencial que cala muy «hondo» en los alumnos y que se evitaría si se suprimieran, del currículo de bachillerato, los procesos ácido-base en los que participan más de un equilibrio y si se pospusieran estos procesos para niveles superiores.

Desde un punto de vista didáctico, sería interesante marcar diferencias en cuanto a los contenidos que aparecen en los libros de educación secundaria y los que aparecen en los universitarios (se puede tomar, como ejemplo, el tratamiento que realizan los libros antiguos), reservando para este nivel los contenidos en los que se ha demostrado la dificultad de aprendizaje para los estudiantes (incluso de los niveles superiores). De este modo, en educación secundaria se tratarían sólo las valoraciones de ácido fuerte con base fuerte y se reservarían las que requieren equilibrios múltiples para los niveles universitarios.
Concepciones alternativas de los licenciados (futuros docentes de educación secundaria) sobre los equilibrios ácido-base múltiples

El protocolo diseñado para el análisis de contenidos puede ser aplicado a cualquier texto escrito, por lo que hemos analizado una muestra aleatoria de 50 exámenes de licenciados que aspiran al cuerpo de profesores de educación secundaria (especialidad física y química) por lo que ignoramos cuáles de ellos ejercen actualmente como profesores (De Manuel et al., 2000). Dichos exámenes corresponden a la prueba escrita de un tribunal en el que fue seleccionado por azar el tema de ácidos y bases (núm. 55 del temario oficial según marca el BOE de 21 de septiembre de 1993) en el que se recogen los siguiente epígrafes que tienen que desarrollar los aspirantes: Ácidos y bases. Teorías. Medidas del $\mathrm{pH}$. Indicadores. Procedimientos para la realización experimental de una curva de valoración ácido-base. Hidrólisis. Soluciones amortiguadoras. Lluvia ácida y contaminación). Los contenidos que se analizan con el protocolo de los textos están incluidos en estos epígrafes, por lo que dicho protocolo puede ser utilizado para el análisis de los exámenes escritos.

Los exámenes constituyen una buena oportunidad para analizar las concepciones de los licenciados porque en ellos van a desarrollar lo más ampliamente posible el tema, ya que, si superan esta prueba, pueden obtener una plaza permanente de profesores de educación secundaria (ipara toda la vida!). Además los aspirantes suelen ser recién licenciados que, por tanto, tienen bastante actualizados sus conocimientos sobre el tema. Ambos factores hay que tenerlos en cuenta porque manifiestan interés y motivación elevados para expresar de la mejor forma posible sus conocimientos sobre este tema y, por tanto, las concepciones alternativas que se expliciten responderán a ideas persistentes y a las inducidas por sus estudios de preparación a estas pruebas selectivas.

El análisis del segundo epígrafe del tema 55 (Procedimiento para la realización experimental de una curva de valoración ácido-base) muestra que el $64 \%$ de los opositores sólo considera una valoración entre un ácido y una base fuertes cuyo punto de equivalencia coincide con un valor del $\mathrm{pH}=7$. El 14\% comenta explícitamente que, en las valoraciones, el punto de equivalencia coincide con un $\mathrm{pH}=7$. Sólo el $12 \%$ tiene en cuenta la valoración de un ácido débil con una base fuerte o viceversa y tan sólo dos opositores $(4 \%)$ se refieren a todas las posibilidades. Casi todos éstos indican que, en el punto de equivalencia, primero se forma la sal y la hidrólisis posterior de ésta produce un $\mathrm{pH} \neq 7$ (razonamiento causal secuencial en el que una etapa es causa de la posterior sin considerar que todos los equilibrios tienen lugar desde el principio).

Como sucede en los alumnos universitarios, los opositores no aplican correctamente dos conceptos relacionados con los indicadores: son pocos $(32 \%)$ los que tienen en cuenta su equilibrio y más de la mitad (52\%) no considera que, en el intervalo de viraje, el color que se aprecia es mezcla de los dos, como se manifiesta en muchas de sus frases: 


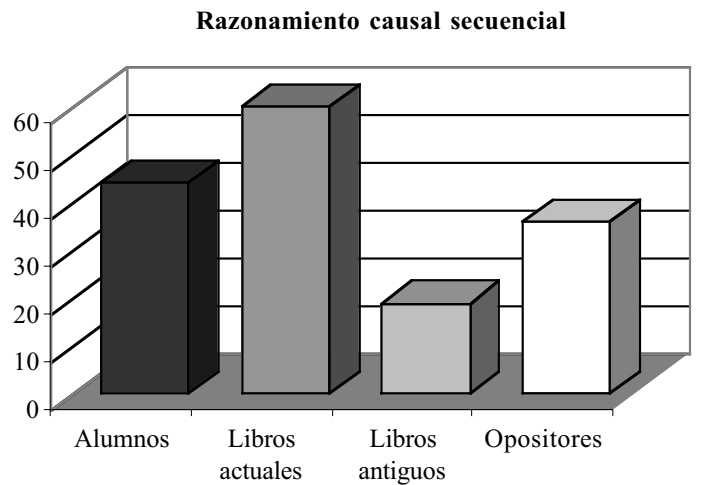

- «Se trata de especies que no intervienen de ninguna forma en la reacción que transcurre [...] se encuentra de forma paralela, es decir, no afecta ni a la velocidad de reacción ni a la formación de unas u otras especies, únicamente tiene como misión indicar el cambio de $\mathrm{pH} . »$

- «Al añadir la base al ácido, éste no irá cambiando progresivamente sino que, cuando la disolución deje de ser ácida, justo en ese momento, cambiará de color el indicador.»

- «Cambio de color brusco en el intervalo de pH.»

- «Justo en el momento en que el indicador cambia de la forma coloreada que tenían a la no coloreada $\Rightarrow \mathrm{PE} . »$

- «En la zona de viraje hay un cambio brusco de pH.»

Como indicamos en párrafos anteriores, algunos opositores no describen ni el equilibrio ni el intervalo de viraje aplicando casi textualmente la definición expresada por Monzón en 1917. Por ejemplo: «[...] el fundamento es que cuando se valora un ácido con una base se produce un salto brusco en el $\mathrm{pH}$; cuando se neutraliza todo el ácido ya no hay $\mathrm{H}_{3} \mathrm{O}^{+}$para neutralizar los $\mathrm{OH}^{-}$, entonces se desplaza el equilibrio de disociación del indicador produciéndose el cambio en la coloración». Las gráficas 1 y 2 ofrecen un resumen de la fase de diagnóstico.

Los tres estudios realizados (alumnos, libros y licenciados) revelan algunas dificultades en el aprendizaje de estos contenidos que pueden solventarse mediante estrategias de enseñanza que incidan directamente sobre las concepciones más destacadas: la ausencia de algunos equilibrios (por ejemplo, el del indicador) en las valoraciones (y en los procesos de equilibrios múltiples), con lo que se le está concediendo más importancia a unos equilibrios frente a otros que son despreciados, y el tratamiento de los equilibrios múltiples como si fueran una secuencia de pasos sucesivos que no transcurre desde el principio ni de forma simultánea.

Como hemos dicho en párrafos anteriores, el origen de ambas concepciones alternativas puede encontrarse en la presentación anticipada de los equilibrios múltiples de la educación secundaria y la necesidad de muchos docentes y autores de libros de tener que recurrir al razonamiento causal secuencial para facilitar su comprensión. Por todo ello, se debería retrasar la presentación a los estudiantes de los equilibrios múltiples hasta que se haya logrado el aprendizaje de los contenidos relacionados con los equilibrios «simples». Además de este retraso en la presentación de los equilibrios múltiples hasta los niveles universitarios, sería necesario mejorar su enseñanza. Para ello, la resolución de problemas ofrece una buena ocasión para que los alumnos utilicen y controlen las variables que intervienen en los procesos ácido-base de varios equilibrios. Sin embargo, antes de utilizar los problemas de equilibrios múltiples debemos analizar si los que se están empleando ayudan a mejorar el aprendizaje de estos contenidos o lo dificultan.

Un buen tratamiento de la resolución de problemas puede facilitar el aprendizaje de los equilibrios múltiples, ya que el planteamiento de problemas semánticamente ricos, donde se incida en la resolución de todas las variables posibles, permitiría evitar que se consideren como compartimentos estanco y se comenzaría a desarrollar la simultaneidad de dichos equilibrios. Con este objetivo, a continuación, vamos a mostrar el análisis de los problemas y de los procesos de resolución que proponen algunos libros universitarios. Los resultados mostrarán la conveniencia de modificar los enunciados y la mejora del proceso de resolución, pues de esos dos factores surgen las posibles soluciones al problema del razonamiento causal secuencial en los equilibrios ácido-base múltiples. 


\section{LA RESOLUCIÓN DE PROBLEMAS SOBRE LOS EQUILIBRIOS ÁCIDO-BASE}

\section{Antecedentes sobre la resolución de problemas}

La definición del término problema abarca muchos significados, desde el sentido estrictamente etimológico de lanzar hacia adelante hasta el significado matemático, que sería una proposición dirigida a averiguar el modo de obtener un resultado cuando ciertos datos son conocidos. Como indica Perales (2000), la didáctica de las ciencias actual tiende a englobar bajo este término toda actividad (ya sea en el aula o en la vida cotidiana) que implica incertidumbre. A diferencia de lo que sucede con los problemas cotidianos en el campo educativo, el planteamiento y la resolución de los problemas son intencionados y con ellos se pretende facilitar el aprendizaje de determinados conceptos y procedimientos. Según este mismo autor, los problemas se pueden clasificar en función del procedimiento seguido para su resolución en: problemas de aplicación directa («ejercicios»); problemas algorítmicos (implican el seguimiento de una secuencia de operaciones cerradas); problemas heurísticos (requieren una estrategia previamente planificada) y problemas creativos (aquéllos que pueden resolverse de diferentes formas sin estrategia predeterminada).

Los problemas de química suelen ser de temática menos rica que los de física, pero más interconectada, ya que hay conceptos centrales como los de mol, estequiometría, etc. que están presentes en casi todas las parcelas de la química (Fernández González, 2000). También suelen ser problemas cerrados (solución única) y cuantitativos (requieren procedimientos gráficos o de cálculo matemático para su resolución).

La resolución de un problema de química requiere que el alumno utilice la información teórica y los procedimientos necesarios así como que posea una actitud favorable hacia la tarea. Por tanto, los objetivos que pueden perseguirse con el uso de la resolución de problemas en la enseñanza están relacionados con el aumento de la capacidad de resolver problemas y con la toma de decisiones, sobre todo en la vida cotidiana, potenciándose la comunicación con otros individuos si la resolución se lleva a cabo de forma colectiva y el aprendizaje de habilidades tanto sensomotoras como cognitivas además de una mejora de las actitudes hacia la ciencia y el conocimiento científico (Perales, 2000).

Los investigadores en didáctica de las ciencias están concediendo una especial importancia al tópico de la resolución de problemas (Hobden, 1998; Sherwood et al., 1998). A pesar de ello, los ejemplos relacionados con el ámbito de la química siguen siendo escasos; podemos citar a Kempa (1986), quien propone un mapa conceptual relacionado con los conceptos de ácidos y de bases para relacionarlo con la información que ofrece el problema y para buscar las causas de la incapacidad que muestran muchos estudiantes a la hora de resolverlos. También encontramos aplicaciones a fin de mejorar el proceso de resolución de algunos conceptos fundamentales de química en un nivel medio (Fernández González, 2000).
Centrándonos en los problemas de química, queremos citar a Niaz (1988, 1989, 1993 entre numerosas investigaciones) por investigar sobre las estrategias de resolución de estos problemas en función del nivel de desarrollo cognitivo y la demanda-M (Pascual-Leone, 1978, 1987), destacando que los estudiantes encuentran más dificultad en la resolución si aumenta la demanda-M de los problemas planteados. Las aportaciones de Niaz (basadas en el análisis dimensional neopiagetiano de Pascual-Leone) permiten evaluar la demanda-M (exigencia, dificultad cognitiva) de los problemas de química.

Centrándonos en los problemas sobre ácidos y bases, Anamuah-Mensah (1986) estudia la estrategia de resolución de 47 alumnos de educación secundaria ante el cálculo de la concentración de ácido clorhídrico, mediante la valoración con hidróxido de sodio, así como la predicción que realizan los alumnos ante lo que sucedería si se cambiara a una estequiometría 2:1, si en vez de $\mathrm{HCl}(\mathrm{ac})$ utilizaran $\mathrm{H}_{2} \mathrm{SO}_{4}(\mathrm{ac})$ o como valorante $\mathrm{Na}_{2} \mathrm{CO}_{3}(\mathrm{ac})$. Según el autor, los alumnos usan básicamente dos estrategias: una basada en las fórmulas del tipo $\mathrm{V} \mathrm{M}=\mathrm{V}$ ' M' o moles = Molaridad Volumen, que es la más frecuente entre los alumnos diagnosticados (79\%); y otra estrategia basada en la proporcionalidad de volúmenes y molaridades. Este último proceso de resolución es utilizado por aquellos alumnos que no suelen recordar las fórmulas, pero que saben utilizar relaciones de proporcionalidad directas. Las dificultades en torno a estos problemas que detecta el autor tienen su origen en la combinación de pasos (escribir balance de masa, estequiometría, cálculo de moles, etc.). El autor anima a los profesores a que propongan estrategias de resolución alternativas a las fórmulas y los algoritmos que, aunque para muchos alumnos resultan más fáciles de utilizar, sin embargo, para otros sería preferible hacerlo usando relaciones entre variables que recordando fórmulas memorizadas.

Boujaoude (1993) estudia los errores sistemáticos de los estudiantes de dicho nivel a la hora de resolver los problemas de equilibrios cinéticos y químicos y concluye que dichos estudiantes no comprenden la relación entre los resultados experimentales y el grado de la reacción, centrándose más en la búsqueda de una solución matemática que en una interpretación química. Por otro lado, Huddle y Pillay (1996), analizando las respuestas de los alumnos en un examen de química general, comprueban que no manejan adecuadamente estos conceptos y consideran que la causa puede ser su alto nivel de abstracción que no se adecua al nivel de desarrollo cognitivo de los alumnos, pues muchos no han alcanzado el nivel de las operaciones formales. Mason y Crawley $(1994,1997)$ comparan el proceso de resolución de expertos (profesores universitarios) y novatos (estudiantes) de algunos problemas algorítmicos y otros conceptuales. Estos autores comentan que los problemas algorítmicos requieren mayor tiempo y mayor número de transiciones que los problemas conceptuales, y que los novatos resuelven los problemas algorítmicos correctamente un mayor número de veces que los expertos. Las diferencias entre los procesos de resolución que muestran los solucionadores expertos y novatos también han sido estudiadas por Kumar (1993) y Kumar y otros (1994). 
De todas las posibilidades que nos ofrece la investigación sobre esta importante actividad en nuestra área, centraremos nuestra atención en la enseñanza de estrategias heurísticas, aunque no descartamos trabajar en el futuro sobre otros modelos de investigación en resolución de problemas (influencia de las variables del problema, resolución por expertos y novatos, adecuación del nivel de exigencia al desarrollo cognitivo de los solucionadores, etc). Nuestra propuesta pretende ofrecer a los alumnos una estrategia que les permita resolver los problemas de equilibrios ácido-base (en los que hay que controlar muchas variables, ya que se deben considerar varios equilibrios simultáneos), sin exponerlos al razonamiento causal secuencial y a otros obstáculos descritos y diagnosticados en alumnos universitarios, como hemos mostrado antes.

Como señala Polya (1945), citado en Perales (2000, p. 37), las estrategias deben adscribirse a las siguientes etapas: definición del problema (selección de la información necesaria), planificación del problema (esquema de resolución), ejecución y retroacción (revisión del proceso).

Encontramos varios ejemplos de propuestas de heurísticas para temas concretos de física. Por citar un ejemplo, Perales (1994) aplicó, con sus alumnos, un método heurístico a fin de resolver problemas de mecánica y, tras la realización de esta investigación, nos indica que resulta difícil romper con la secuencia típica de los alumnos para solucionar problemas (datos $\rightarrow$ fórmulas $\rightarrow$ resultado), que no todas las etapas de la heurística propuesta eran utilizadas por igual o que la repetición del método heurístico por parte de los alumnos ocasiona que se centren en algunas etapas.

\section{Análisis de los problemas de equilibrios ácido-base}

La temática en los problemas de química está asociada al grado de dificultad: los problemas de estequiometría no suelen tener el mismo grado de dificultad que los que tratan sobre equilibrios químicos; por otro lado, también se producen diferencias en el proceso de resolución (Fernández González, 2000). Este autor comenta algunas características de los problemas de equilibrio químico para el nivel de educación secundaria e indica que se trata de un concepto abstracto que ofrece serias dificultades para su comprensión, ya que implica dos procesos contrarios y simultáneos.

En este nivel sólo se abordan los problemas en los que aparece un equilibrio únicamente. En el nivel universitario aumenta la complejidad cognitiva ${ }^{1}$ al aumentar el número de equilibrios que hay que considerar en un mismo proceso. Como hemos indicado en párrafos anteriores, los alumnos universitarios no consideran (ni los licenciados describen) todos los equilibrios presentes en un proceso, lo hacen «por compartimentos estanco» o mediante el razonamiento causal secuencial ya expuesto.

Por otro lado, los problemas de equilibrios múltiples, en concreto los que tienen lugar en un proceso ácido-base, poseen una complejidad matemática añadida. Se suelen delimitar casos particulares (ácidos fuertes - bases fuertes, ácidos fuertes - bases débiles, etc.) y, además, se pueden utilizar procedimientos aproximados en los que se prescinde de parte de los equilibrios.

Al realizar nuestra propuesta de resolución de los problemas con esta temática, debemos evitar el empleo de fórmulas algebraicas que sólo conducirían a un operativismo mecánico y alejarían al alumno de una adecuada utilización de los conceptos. Nuestra propuesta debe basarse fundamentalmente en eliminar el formulismo matemático (tarea que se sustituye por la utilización de un programa informático) para centrar la atención del alumno en todos los equilibrios presentes en el proceso ácido-base y eliminar los problemas-tipo. Se trataría de ampliar el abanico de posibilidades a todas las combinaciones entre ácidos y bases (dos ácidos monopróticos, polipróticos, mezclas de ácidos y de bases, hidrólisis de sales considerándolas como un proceso ácido-base más, sin tener que referirnos a ella de forma explícita, etc.).

Siguiendo con la metodología utilizada para analizar los contenidos que aparecen en los libros de texto, hemos diseñado un breve protocolo para cuantificar de manera objetiva algunos aspectos de los problemas propuestos en los libros de texto, y los agruparemos. Con este fin, hemos seleccionado tres libros universitarios porque ofrecen abundantes enunciados de problemas $(\mathrm{N}=574)$ sobre esta temática (uno de química analítica y dos de química general, uno de los cuales es específico de problemas). En los dos libros de química general se resuelven algunos de los problemas planteados, por lo que hemos podido analizar este proceso.

El protocolo diseñado sirve para indagar sobre aspectos descriptivos: cuantificar el número de problemas que son algorítmicos, heurísticos o creativos; y clasificar los «tipos de problemas» que plantean los libros para explorar si tienden a generalizar o, por el contrario, a crear parcelas o estereotipos de problemas que los alumnos deben saber resolver «estudiando» el proceso que sigue el libro o el profesor.

Un aspecto que consideramos importante en nuestro estudio es comprobar si, en la resolución que proponen los libros seleccionados, se pone excesivo énfasis en el cálculo matemático y, por tanto, en el resultado numérico o si, por el contrario, se insiste más en aspectos cualitativos como, por ejemplo, en la discusión de los resultados. Dicho énfasis matemático puede estar relacionado con el número de aproximaciones que realicen, por lo que las cuantificaremos. Los ítems que aplicamos a la muestra de problemas se expresan en la tabla II.

\section{Resultados de la aplicación del protocolo}

En la tabla III exponemos los resultados obtenidos tras el análisis de los problemas en los tres libros seleccionados mediante la aplicación de los tres primeros ítems del protocolo anterior, mientras que en la tabla IV mostramos los resultados de los ítems 4 y 5 correspondientes al proceso de resolución de los problemas de los libros. 
Tabla II

Protocolo para el análisis de problemas.

\begin{tabular}{|c|c|}
\hline Contenidos & Posibles resultados \\
\hline $\begin{array}{l}\text { Ítem 1. } \\
\text { 1.1. ¿Cuántos son cuestiones teóricas? } \\
\text { 1.2. ¿Cuántos son ejercicios? } \\
\text { 1.3. ¿Cuántos son algorítmicos? } \\
\text { 1.4. ¿Y cuántos heurísticos? } \\
\text { 1.5. ¿Y creativos? }\end{array}$ & $\begin{array}{l}\text { En este ítem contamos los problemas que corresponden a la } \\
\text { clasificación mencionada y explicada por Perales }(2000) \text {. }\end{array}$ \\
\hline $\begin{array}{l}\text { Ítem } 2 . \\
\text { Categorización de los problemas según la temática. } \\
\text { ¿Qué temática tiene mayor número de problemas? }\end{array}$ & $\begin{array}{l}\text { Mediante este ítem clasificamos los problemas. Por otro lado, contamos } \\
\text { los problemas que puedan ser introducidos en cada categoría y de este } \\
\text { modo comprobamos a qué tipo de problemas se concede mayor } \\
\text { importancia. }\end{array}$ \\
\hline $\begin{array}{l}\text { Ítem } 3 . \\
\text { ¿Reducen los problemas a la búsqueda de una sola incógnita, } \\
\text { por ejemplo, el pH? }\end{array}$ & $\begin{array}{l}\text { Este ítem sirve para examinar si se concede importancia exclusivamente } \\
\text { a una de las especies: }\left[\mathrm{H}_{3} \mathrm{O}^{+}\right] \text {o si, por el contrario, también se insiste } \\
\text { en la búsqueda de las concentraciones de las demás especies. }\end{array}$ \\
\hline $\begin{array}{l}\text { Ítem } 4 \text {. } \\
\text { ¿Consideran todos los equilibrios presentes en el proceso o, por el } \\
\text { contrario, los reducen a los que interesan para llegar al resultado del } \\
\text { problema? }\end{array}$ & $\begin{array}{l}\text { El objetivo de este ítem es contar el número de equilibrios totales y } \\
\text { compararlo con el número de equilibrios que tienen en cuenta en el } \\
\text { proceso de resolución. }\end{array}$ \\
\hline $\begin{array}{l}\text { Ítem } 5 . \\
\text { ¿Muestran un excesivo énfasis en el cálculo matemático? }\end{array}$ & $\begin{array}{l}\text { Indagamos sobre el número de aproximaciones que realizan, así como } \\
\text { la relación de dichas aproximaciones con la discusión cualitativa, tanto } \\
\text { de las aproximaciones como de los resultados obtenidos. }\end{array}$ \\
\hline
\end{tabular}

El número de problemas que aparecen en la clasificación por temas (ítem 2) supera el número total de problemas que propone el libro. Eso es debido a que esta clasificación no es excluyente, ya que un mismo problema puede estar incluido en el apartado del $\mathrm{pH}$ y en el de $\mathrm{K}_{\mathrm{a}}$ si en él se solicitan estas dos incógnitas.

La segunda parte del protocolo (ítems 4 y 5) muestra las pautas para el análisis del proceso de resolución desarrollado por los libros de química general (QG) y de problemas (QGP). No incluimos ningún problema del libro de química analítica porque en él sólo se plantean enunciados y no se desarrolla la resolución de ninguno de ellos. En la tabla IV aparecen los resultados de este análisis para los 8 problemas propuestos que aparecen numerados seguidos del libro al que pertenecen.

\section{Análisis de los resultados}

Atendiendo a la clasificación que exponemos en la tabla III observamos que los problemas que proponen los libros sobre los ácidos y las bases no son creativos (donde el alumno debe plantear su propia estrategia) y tampoco pueden considerarse algorítmicos, ya que no hay una estrategia de pasos cerrada, como, por ejemplo, en el caso del ajuste de las ecuaciones «redox». La mayoría de los problemas son de aplicación directa o ejercicios en los que se solicita al alumno que determine una incógnita mediante operaciones simples. Aunque en la tabla no aparezca explícitamente, la mayoría de estos problemas tienen varios subproblemas que, a su vez, son ejercicios.
En la citada tabla podemos observar que escasean los problemas heurísticos, entre los que hemos incluido los que plantean varios equilibrios simultáneos y, por tanto, requieren una estrategia planificada para su resolución. Este hecho resulta sorprendente, pues en la sección de los libros destinada a la exposición de los contenidos teóricos, previa a los problemas, se profundiza en los equilibrios múltiples y, sin embargo, se proponen muy pocos problemas para aplicar estos conceptos.

Otro dato sobre el que queremos llamar la atención es que, como era de esperar, el libro de problemas no plantea ninguna cuestión teórica, ya que hace una breve exposición teórica o resumen de otro libro. Sin embargo, el otro texto de química general sí plantea abundantes cuestiones teóricas y en el libro de química analítica analizado también se proponen algunas. Estas cuestiones pueden ser cualitativas, como por ejemplo, la identificación de sustancias ácidas o básicas, o también cuantitativas, como sería la comparación de la fuerza de dos ácidos utilizando las constantes de equilibrio.

Con respecto a la temática, ningún libro plantea problemas de la vida cotidiana, aunque, como hemos comentado antes, alguno utiliza productos cotidianos para plantear los mismos problemas que con productos exclusivos del contexto científico. El tema que recibe mayor protagonismo es la determinación del $\mathrm{pH}$, aunque en el libro de química analítica se solicitan mayoritariamente las concentraciones de las especies en los problemas que plantea. También observamos que suelen proponerse problemas de disolu- 
Tabla III

Clasificación de los problemas.

\begin{tabular}{|c|c|c|c|}
\hline Clasificación & $\begin{array}{l}\text { Libro de química } \\
\text { general }(\mathrm{N}=158)\end{array}$ & $\begin{array}{c}\text { Libro de } \\
\text { problemas }(N=40)\end{array}$ & $\begin{array}{l}\text { Libro de química } \\
\text { analítica }(N=16)\end{array}$ \\
\hline $\begin{array}{l}\text { Ítem } 1 . \\
\text { Según el procedimiento: } \\
\text { 1.1. Cuestiones teóricas } \\
\text { 1.2. Ejercicios } \\
\text { 1.3. Algorítmicos } \\
\text { 1.4. Heurísticos } \\
\text { 1.5. Creativos }\end{array}$ & $\begin{array}{l}61(38 \%) \\
83(52 \%) \\
0 \\
15(10 \%) \\
0\end{array}$ & $\begin{array}{l}0 \\
29(73 \%) \\
0 \\
11(27 \%) \\
0\end{array}$ & $\begin{array}{l}5(31 \%) \\
6(38 \%) \\
0 \\
5(31 \%) \\
0\end{array}$ \\
\hline $\begin{array}{l}\text { Ítem } 2 . \\
\text { Según la temática: } \\
\text { 2.1. Naturaleza ácido-base (identificación de } \\
\text { sustancias, propiedades, fortaleza, etc.) } \\
\text { 2.2. pH } \\
\text { 2.3. Concentración de especies }\left(\mathrm{C}_{\text {especies }}\right) \\
\text { 2.4. } \mathrm{K}_{\mathrm{a}}\left(\mathrm{o} \mathrm{K}_{\mathrm{b}}\right) \\
\text { 2.5. Cantidad de sustancia } \\
\text { 2.6. Volumen de agente valorante } \\
\text { 2.7. Tampones } \\
\text { 2.8. Titulaciones ácido-base } \\
\text { 2.9. Cotidianos }\end{array}$ & $\begin{array}{l}42 * \\
67 \\
15 \\
16 \\
3 \\
1 \\
35 \\
15 \\
0 * *\end{array}$ & $\begin{array}{l}0 \\
24 \\
9 \\
7 \\
0 \\
0 \\
2 \\
5 \\
0\end{array}$ & $\begin{array}{l}2 \\
4 \\
5 \\
0 \\
0 \\
0 \\
1 \\
1 \\
0\end{array}$ \\
\hline $\begin{array}{l}\text { Ítem } 3 . \\
\text { Según el número de incógnitas: } \\
\text { 3.1. Una sola incógnita }\left(\mathrm{pH}, \mathrm{C}_{\mathrm{a}},\left[\mathrm{H}^{+}\right], \mathrm{K}_{\mathrm{a}} \text {, etc.) }\right. \\
\text { 3.2. Dos incógnitas }\left(\mathrm{pH} \text { y } \mathrm{C}_{\mathrm{a}}, \mathrm{pH} \text { y } \mathrm{pK}_{\mathrm{a}} \text {, etc. }\right) \\
\text { 3.3. Tres o más incógnitas }\left(\mathrm{C}_{\text {especies }}, \mathrm{pH} \text { y } \mathrm{C}_{\text {especies }} \text {, etc. }\right)\end{array}$ & $\begin{array}{l}75(47 \%) \\
13(8 \%) \\
12(8 \%)\end{array}$ & $\begin{array}{l}29(73 \%) \\
6(15 \%) \\
5(12 \%)\end{array}$ & $\begin{array}{l}10(63 \%) \\
0 \\
1(6 \%)\end{array}$ \\
\hline
\end{tabular}

* En este ítem, las categorías no son excluyentes. Un mismo problema puede ser clasificado en dos categorías; por ejemplo, en la del pH y en la de la determinación de concentraciones de especies porque haga referencia explícita a ambas variables.

** Algunos problemas utilizan productos comunes y conocidos por los estudiantes como, por ejemplo, la aspirina, la sangre, los refrescos, la cafeína, etc. Sin embargo, no podemos considerarlos como problemas cotidianos, ya que no plantean situaciones de la vida diaria que puedan ser resueltas mediante la aplicación de conceptos científicos.

Tabla IV

Resultados del análisis del proceso de resolución de los problemas.

\begin{tabular}{|c|c|c|c|c|c|}
\hline Problema & $\begin{array}{l}\text { Equilibrios } \\
\text { totales }\end{array}$ & $\begin{array}{l}\text { Equilibrios utilizados } \\
\text { (y porcentaje) }\end{array}$ & $\begin{array}{l}\text { Num. de aproximaciones } \\
\text { utilizadas (y justificadas) }\end{array}$ & $\begin{array}{l}\text { Resultados } \\
\text { numéricos }\end{array}$ & $\begin{array}{c}\text { Valorados } \\
\text { cualitativamente }\end{array}$ \\
\hline 1-QGP & 4 & $1(25 \%)$ & $1(1)$ & Sí & Sí \\
\hline 2-QGP & 4 & $2(50 \%) *$ & $2(2)$ & Sí & No \\
\hline 3-QGP & 3 & $2(66 \%)$ & $1(1)$ & Sí & No \\
\hline 4-QGP & 5 & $1(20 \%)$ & $1(1)$ & Sí & No \\
\hline 5-QG & 6 & $2(33 \%)$ & 0 & No & Sí \\
\hline 6-QG & 3 & $1(33 \%)$ & $1(1)$ & Sí & Sí \\
\hline 7-QG & 4 & $2(50 \%)$ & $1(0)$ & Sí & No \\
\hline 8-QG & 5 & $2(40 \%)$ & 0 & Sí & No \\
\hline
\end{tabular}

* En este problema, el autor del libro utiliza dos equilibrios aunque uno de ellos no considera que el proceso sea reversible, sino como una reacción en un solo sentido. 
ciones reguladoras y de valoraciones ácido-base; sin embargo, no suelen plantearse de una manera explícita problemas de cálculo de volúmenes y son escasos los que se centran en la determinación de la cantidad de sustancia. Tal vez estos últimos problemas (cantidad de sustancia) se reserven para el tema de la estequiometría de las reacciones sin tener en cuenta, por ejemplo, que es el factor principal de las valoraciones.

Por último, la mayoría de los problemas piden el cálculo de una sola incógnita, lo que manifiesta que no aprovechan al máximo las posibilidades de los equilibrios, ya que los reducen a la determinación del $\mathrm{pH}$, de la constante de equilibrio o de la concentración de una especie, pudiendo, sin embargo, solicitar el mayor número posible de incógnitas, por ejemplo, las concentraciones de todas las especies que participan en el proceso. Nos llama la atención que en el libro de problemas sí se demanda el cálculo de más de dos especies y, de forma contraria, es llamativo que en el libro de química analítica no se incida en este aspecto, sobre todo después de insistir en ello a lo largo del tratamiento teórico.

Uno de los objetivos que nos planteamos al diseñar los ítems sobre el proceso de resolución era indagar si los libros emplean todos los equilibrios presentes en el proceso o sólo utilizan los que convenían para llegar por aproximación al resultado del problema. Los resultados de la tabla IV muestran que los libros reducen drásticamente los equilibrios posibles, utilizando únicamente los que interesan para sus fines sin justificar esta reducción. Este resultado nos confirma que los libros universitarios no insisten en el uso de todos los equilibrios, lo que puede reforzar o inducir el razonamiento causal secuencial o también la consecuencia de que los procesos transcurren en una «dirección preferida» (irreversibilidad).

Al contabilizar el número de aproximaciones matemáticas que utilizan los libros, queríamos analizar si se centraban en el cálculo matemático o, por el contrario, el resultado numérico era lo de menos. Los resultados muestran que en la mayoría de los problemas se realizan aproximaciones matemáticas con el fin de obtener un resultado numérico, con el consiguiente menosprecio de los aspectos químicos. Este dato, unido a que la mayoría de los problemas llegan a un resultado numérico (90\%) sin discutir cualitativamente dicho resultado $(60 \%)$, manifiesta que los autores de los libros ponen excesivo énfasis en el cálculo matemático, menoscabando los aspectos cualitativos, es decir, se insiste en lo matemático frente a lo conceptualmente químico.

Estos resultados nos hacen reflexionar sobre algunos aspectos que debemos considerar en la propuesta que vamos a plantear a continuación. En primer lugar, debemos proponer problemas heurísticos con temática diversa, de forma que no centremos la atención sobre el cálculo del $\mathrm{pH}$ o de la concentración de iones $\mathrm{H}_{3} \mathrm{O}^{+}$, sino que insistamos por igual en todas las especies presentes en el proceso. Esta insistencia unida a la de utilizar todos los equilibrios posibles ayudará a una mayor comprensión del problema «químico», relegando a un segundo plano el «problema matemático».

\section{El problema de los equilibrios múltiples: el ordenador como recurso auxiliar para su resolución}

Las dificultades encontradas (excesivo énfasis en el cálculo matemático y amplia reducción del número de equilibrios considerados) nos hacen reflexionar sobre la necesidad de incorporar el uso del ordenador para reducir a un papel secundario el cálculo matemático en la enseñanza de estos problemas y, de este modo, centrar la atención del alumno en la comprensión y la utilización de todos los equilibrios presentes en el proceso.

Como indica Jonassen (1999), el ordenador, utilizado como herramienta cognitiva, puede ayudar a los alumnos como suplemento a su memoria haciendo disponible gran cantidad de información de manera inmediata. También puede simultanear (o recuperar cuando sea preciso) la información relevante aprendida con anterioridad a la adquirida en la actualidad. Esto permite a los alumnos que organicen y relacionen sus ideas previas con las nuevas, así como facilitarles la autocomprobación y la representación de ideas tanto verbal como pictóricamente.

Sierra (2000) indica que algunas tareas cognitivas de la enseñanza de las ciencias pueden ser facilitadas por el ordenador, como, por ejemplo: soportar procesos cognitivos (memorización, acceso al conocimiento declarativo y conceptual), ya que el ordenador puede hacer un seguimiento y registrar los pasos dados por el alumno al resolver un determinado problema; compartir la carga cognitiva, en nuestra propuesta consistiría en ayudar en el proceso matemático permitiendo que los alumnos se centren en las tareas correspondientes al ámbito de la química y, en concreto, de los equilibrios ácido-base; estimula al alumno en las actividades cognitivas, ya que el ordenador acerca el mundo real de forma concreta y manipulable; y, por último, permite al alumno generar y probar hipótesis en el contexto de la resolución de problemas.

Según Zabalza (1985) el ordenador posee varias funciones que repercuten de manera significativa en la educación. Estas funciones son las siguientes: innovadora (nuevo tipo de interacción); motivadora (aproxima la realidad y diversifica las formas de acceso a ella); estructuradora de la realidad (interpreta la realidad y se la transmite al alumno); mediatizadora (establece un tipo de relación con el alumno que condiciona las operaciones mentales que se promueven); operativa (facilita y organiza las acciones de los alumnos); y formativa global (crea su propio espacio didáctico). En nuestra propuesta vamos a utilizar el ordenador básicamente en su función operativa, pero de forma no explícita también utilizaremos las otras descritas por Zabalza.

Como muestras de utilización del ordenador para el área de química citaremos, el trabajo de Vidal de Labra y otros (1985), quienes propusieron actividades de ejercitación y práctica para el caso concreto de las disoluciones y sus concentraciones así como el de Blondel y Schwob (1996), los cuales de forma interactiva plantean la resolución de problemas de química a partir de una base de datos y un sistema experto. 
Por todo esto, la utilización del ordenador como ayuda en la resolución de los problemas de equilibrios múltiples pretende no sólo centrar la atención sobre los contenidos químicos, posibilitando la consideración de todos los equilibrios, sino también disminuir con ello el riesgo de caer en el razonamiento causal secuencial o en considerar los equilibrios como «compartimentos estanco».

\section{Propuesta de actividades que se pueden aplicar en el aula}

Las propuestas de actividades, diseñada para alumnos universitarios, se centran en varios aspectos:

1) Dotar a los alumnos de un método heurístico para evitar la resolución de forma mecánica e irreflexiva de los problemas de equilibrios múltiples. Por ejemplo, Perales (1994) realiza una propuesta de utilización en el aula de esta técnica, planteando una heurística basada en cinco etapas: definición (información pertinente), planificación (esquema de la solución), ejecución (solución provisional), vuelta al comienzo de la resolución y, por último, la solución definitiva. Los estudiantes universitarios pueden manejar este método en problemas sencillos.

2) En esta etapa el docente propone varios problemas de equilibrios múltiples sencillos para que los alumnos se familiaricen con la explicitación de todos los equilibrios que intervienen en el proceso. No es necesario resolver todo el problema, tan sólo se realiza una valoración cualitativa del problema químico, proponiéndose la solución aproximada (carácter ácido-base de la solución final, etc.).

3) Tratamiento de los problemas de equilibrio ácido-base utilizando todas las incógnitas posibles y estableciendo ecuaciones con las constantes de equilibrio y las definiciones de las diferentes concentraciones analíticas.

De esta forma, el aspecto químico del problema queda bien planteado, se tienen en cuenta todos los equilibrios, y se ha reducido al aspecto matemático del problema, es decir, hay que encontrar las soluciones a las incógnitas. Podríamos decir que hemos concluido con el aspecto químico (aunque faltaría interpretar o discutir los resultados) y, por tanto, sólo faltaría buscar estrategias o caminos que permitan una resolución lo más simple posible del problema matemático.

Sin salvar las dificultades matemáticas, los alumnos pueden continuar ejercitando la obtención de ecuaciones para calcular otras especies y otros problemas: bases monobásicas; mezclas de dos, tres... $n$ ácidos monopróticos; mezclas de dos, tres... $n$ bases monobásicas; ácidos polipróticos; bases polibásicas.

4) Tras comprobar que se obtienen ecuaciones con grados cada vez mayores, se pueden plantear dos opciones: por un lado, simplificar eliminando algunas ecuaciones, con lo que se somete el problema químico a la resolución satisfactoria de un problema matemático o, por otro lado, utilizar un instrumento auxiliar que nos permita salvar esta dificultad sin necesidad de tener que realizar aproximaciones que alejen al alumno de los contenidos químicos implicados (equilibrios, especies químicas, etc.). Con este fin se procederá a la utilización de programas de ordenador específicos sobre los que descargar los cálculos matemáticos difíciles de realizar para los alumnos.

Sin embargo, podemos dar un paso más y utilizar el ordenador para que el alumno interactúe, de forma que en una primera etapa solicite al alumno el número de equilibrios que tienen lugar en el proceso y se establezca una relación con el ordenador de forma que éste pueda responderle si es correcto o no dicho número. Este proceso permite centrar al alumno en los equilibrios químicos y contrastar el estudio cualitativo que ha realizado en etapas previas de esta propuesta de actividades.

Una vez acordado, entre el alumno y el ordenador, el número de equilibrios, el programa solicita al alumno que realice la valoración cualitativa mientras realiza los cálculos numéricos para poder contrastar la solución provisional propuesta por el alumno y la definitiva que plantea el ordenador. De esta forma se insiste en la necesidad de hacer un análisis cualitativo previo y posterior a la obtención del resultado.

El docente puede establecer un debate en pequeños grupos tras el análisis de los resultados obtenidos (provisionales y definitivos), así como de todo el proceso de resolución del problema.

5) Aplicación de la metodología a varios casos concretos. Se plantearán problemas con mayor número de equilibrios (y, por tanto, de variables a considerar), que requieren un tratamiento similar al de ácidos monopróticos, bases monobásicas, ácidos polipróticos y bases polibásicas con ecuaciones más generales que, igualmente, se resuelven con la ayuda del ordenador. Los casos concretos serán:

- Mezclas de ácidos y bases: ácido fuerte - base fuerte; ácido fuerte - base débil; ácido débil - base fuerte; ácido débil - base débil

- Mezclas de ácidos polipróticos (fuertes o débiles) con bases polibásicas (fuertes o débiles).

- La hidrólisis de sales que puede plantearse simplemente como un problema de ácido o de base sencillo para unos determinados iones $u$ otros iones con más cargas pero también pueden plantearse como problema de mezclas de ácidos y de bases. Las disoluciones reguladoras también pueden tratarse como mezclas de ácidos y de bases.

- Los indicadores ácido-base que pueden considerarse como ácidos o bases en el caso de que estén solos o si intervienen en otros procesos, aunque sea en pequeñas cantidades, también habrá que considerarlos como si se tratara una mezcla de ácidos.

- Por último, el problema de obtener los valores del pH durante el transcurso de una volumetría ácido-base es el mismo que el planteado para una mezcla de ácido y base, sólo que repetido para diferentes mezclas que cubren diferentes zonas (o etapas) de la valoración. En estos problemas, un programa de simulación del ordenador permite observar, a tiempo real, la gráfica $\mathrm{pH}$ versus $\mathrm{ml}$ de valorante 
Tabla V

Problemas concretos y objetivos que se pretenden conseguir.

\begin{tabular}{|c|c|}
\hline Objetivos & Problemas \\
\hline $\begin{array}{l}\text { - Consideración de todos los equilibrios. } \\
\text { - Determinación de todas las especies sin conceder excesiva } \\
\text { (o única) importancia a }\left[\mathrm{H}_{3} \mathrm{O}^{+}\right] \text {y al } \mathrm{pH} \text {. } \\
\text { - Los equilibrios múltiples tienen lugar simultáneamente } \\
\text { en todo el proceso. }\end{array}$ & $\begin{array}{l}\text { El ácido acético } 10^{-3} \mathrm{M} \text {, ¿se puede considerar totalmente disociado? Calcule el } \\
\text { grado de disociación a esta concentración }\left(\mathrm{K}_{\mathrm{a}}=1,8 \cdot 10^{-5}\right) \text {. Calcule el } \mathrm{pH} \text { y las } \\
\text { concentraciones de todas las especies iónicas presentes en la disolución. } \\
\text { En la resolución del problema anterior, ¿ha considerado usted la disociación del } \\
\text { agua? Si no lo hizo, hágalo ahora y compare los resultados obtenidos con los } \\
\text { anteriores }\left(\mathrm{K}_{\mathrm{w}}=10^{-14}\right) \text {. }\end{array}$ \\
\hline $\begin{array}{l}\text { - Determinación de todas las especies sin conceder excesiva } \\
\text { (o única) importancia a }\left[\mathrm{H}_{3} \mathrm{O}^{+}\right] \text {y al } \mathrm{pH} \text {. } \\
\text { - Utilización de ácidos polipróticos y bases polibásicas para } \\
\text { la familiarización de los equilibrios múltiples. } \\
\text { - Planteamiento de problemas de mezclas de ácidos, } \\
\text { mezclas de bases, mezclas de ácidos y de bases, etc. } \\
\text { - Los equilibrios múltiples tienen lugar simultáneamente } \\
\text { en todo el proceso. }\end{array}$ & $\begin{array}{l}\text { Calcule el } \mathrm{pH} \text { y las fracciones molares de todas las especies químicas existentes } \\
\text { en el equilibrio para una disolución } 0,5 \mathrm{M} \text { de ácido oxálico. } \\
\text { El ácido etilendiamino tetraacético (EDTA) es un ácido tetraprótico. Calcule las } \\
\text { fracciones molares de todas las especies a los siguientes valores del } \mathrm{pH}: 1,3,7,9 \text {, } \\
12,13 \text { y } 14 \text {. Exprese todos los equilibrios que intervienen, incluido el del agua. } \\
\text { Calcule el pH y las fracciones molares de todas las especies químicas de una } \\
\text { disolución que es } 10-7 \mathrm{M} \text { en } \mathrm{HCl}(\mathrm{ac}) \text { y } 10-4 \text { en ácido acético. }\end{array}$ \\
\hline $\begin{array}{l}\text { - La hidrólisis de la sal como mezclas de ácidos y de bases. } \\
\text { - Solicitar al alumno que resuelva algunos problemas de } \\
\text { dos formas diferentes puede ayudarle a ampliar los } \\
\text { contenidos que tiene que manejar así como los } \\
\text { procedimientos. }\end{array}$ & $\begin{array}{l}\text { Indique dos formas de plantear la resolución de los siguientes problemas y } \\
\text { resolverlos: } \\
\text { a) Calcule las fracciones molares de todas las especies químicas de una disolución } \\
\text { de } 70 \mathrm{~g} \text { de acetato de sodio en } 400 \mathrm{ml} \text { de disolución acuosa. Calcule el } \mathrm{pH} \text {. } \\
\text { b) Calcule el pH correspondiente y las fracciones molares de todas las especies de } \\
\text { una disolución de } 48 \mathrm{~g} \text { de cloruro de amonio en } 250 \mathrm{ml} \text { de disolución acuosa. }\end{array}$ \\
\hline $\begin{array}{l}\text { - Las disoluciones reguladoras no siempre tienen que } \\
\text { elaborarse a partir de cantidades equimolares de sus } \\
\text { componentes. } \\
\text { - Conservación de la cantidad de sustancia en los procesos } \\
\text { de dilución. } \\
\text {-Tarea para resolver un problema sin datos, abierto. }\end{array}$ & $\begin{array}{l}\text { Calcule el } \mathrm{pH} \text { de la disolución reguladora del } \mathrm{pH} \text { que es mezcla de } 0,4 \text { moles de } \\
\text { acetato de sodio y } 0,6 \text { moles de ácido acético en } 100 \mathrm{ml} \text { de disolución. Si sobre } 10 \\
\text { ml de dicha disolución se agrega } 1 \mathrm{ml} \text { de ácido clorhídrico } 0,1 \mathrm{M} \text { o de hidróxido } \\
\text { de sodio } 0,1 \mathrm{M} \text {, ¿cuánto valdrá el } \mathrm{pH} \text { de las disoluciones resultantes? ¿Cuánto } \\
\text { valdrá el pH de las disoluciones resultantes de agregar estas cantidades de ácido o } \\
\text { de base a } 10 \mathrm{ml} \text { de agua? } \\
\text { Diseñe una disolución reguladora de ácido acético y de acetato de sodio que va a } \\
\text { sufrir la adición de una gran cantidad de base. Indique qué considera una «gran } \\
\text { cantidad de base». }\end{array}$ \\
\hline $\begin{array}{l}\text { - Los indicadores poseen un intervalo de viraje y el color } \\
\text { que se aprecia es mezcla de los colores de las dos formas } \\
\text { del indicador. } \\
\text { - El equilibrio del indicador está presente durante todo el } \\
\text { proceso en el que interviene. }\end{array}$ & $\begin{array}{l}\text { Calcule las fracciones molares de cada una de las formas del verde de bromocresol } \\
\text { en función del pH y represente gráficamente su variación. Discuta sobre el color } \\
\text { que se aprecia en cada caso. } \\
\text { Repita el ejercicio anterior para la fenolftaleína. } \\
\text { De los dos indicadores anteriores, ¿cuál sería más adecuado para apreciar cambios } \\
\text { de color en las proximidades del punto de equivalencia de las valoraciones de } \\
\mathrm{CH}_{3} \mathrm{COOH}(\mathrm{ac}) \text { y } \mathrm{HCl}(\mathrm{ac}) \text { con } \mathrm{NaOH}(\mathrm{ac}) \text { y de las valoraciones de } \mathrm{NH}_{3}(\mathrm{ac}) \text { y } \\
\mathrm{NaOH}(\mathrm{ac}) \text { con } \mathrm{HCl}(\mathrm{ac}) \text { ? }\end{array}$ \\
\hline $\begin{array}{l}\text { - Conservación de la cantidad de sustancia en los procesos } \\
\text { de dilución. } \\
\text { - La cantidad de sustancia es la variable que hay que } \\
\text { controlar tanto en las valoraciones como en la adición de } \\
\text { ácidos y de bases a las disoluciones reguladoras. } \\
\text { - Insistir en la determinación de la cantidad de sustancia } \\
\text { en las valoraciones puede ayudar a controlar la operación } \\
\text { de conservación de dicha cantidad de sustancia. }\end{array}$ & $\begin{array}{l}\text { De una muestra de sosa carbonatada se pesan } 2,0658 \mathrm{~g} \text { y se disuelven en agua, } \\
\text { enrasando finalmente a } 500 \mathrm{ml}, 20 \mathrm{ml} \text { de esta disolución valorados con } \mathrm{HCl}(\mathrm{ac}) \\
0,1024 \mathrm{M} \text {, en presencia de fenolftaleína, consumen } 16,3 \mathrm{ml} \text {. Otros } 20 \mathrm{ml} \text {, valorados } \\
\text { en presencia de verde de bromocresol, consumen } 19,2 \mathrm{ml} \text { del ácido. Calcular los } \\
\text { procentajes de } \mathrm{Na}_{2} \mathrm{CO}_{3}(\mathrm{ac}) \text {, de } \mathrm{NaOH}(\mathrm{ac}) \text { y de otras impurezas no valoradas. }\end{array}$ \\
\hline $\begin{array}{l}\text { - Utilización de fenómenos cotidianos para plantear } \\
\text { problemas creativos. }\end{array}$ & $\begin{array}{l}\text { ¿Por qué se previenen las caries con pastas de dientes fluoradas? ¿Sería aconsejable } \\
\text { cepillarse los dientes con } \mathrm{NaHCO}_{3} \text { ? } \\
\text { - En las etiquetas de los envases de sales de fruta se indica que poseen ácido } \\
\text { cítrico, bicarbonato sódico y carbonato sódico. ¿Qué ocurre al usarlo? ¿Por qué se } \\
\text { utiliza esta combinación para combatir la acidez de estómago? ¿No sería mejor } \\
\text { añadir solamente bases? } \\
\text { - Una «receta de la abuela» para la acidez de estómago recomienda tomar zumo }\end{array}$ \\
\hline $\begin{array}{l}\text { NOTA } \\
{ }^{1} \text { El pH del suelo se determina tomando una muestra secada y } \\
\text { tamizada con un tamiz de } 2 \mathrm{~mm} \text { (límite que se considera para el } \\
\text { tamaño del grano de arena); se pesan } 10 \mathrm{~g} \text { de esta muestra y se le } \\
\text { añaden } 25 \mathrm{ml} \text { de agua destilada, se agita durante } 5 \text { minutos y se } \\
\text { deja en reposo media hora, midiéndose el pH con un } \\
\text { «pehachímetro». }\end{array}$ & $\begin{array}{l}\text { bebida? ¿Qué detalles añadiría a esta receta? } \\
\text { - Al fabricar una mermelada de ciruelas rojas, notamos que la mermelada nos está á } \\
\text { quedando muy ácida. Al añadir } \mathrm{NaHCO}_{3} \text { para reducir esa acidez, notamos que el } \\
\text { color de las ciruelas cambia a verdosas. ¿Qué está sucediendo? ¿Seguirán las } \\
\text { ciruelas con ese color o volverán a su color rojizo? } \\
\text { - ¿Qué sustancias debe contener un suelo para que sea ácido? ¿Y básico? En } \\
\text { edafología se emplea el término } \mathrm{pH} \text { del suelo }{ }^{1} \text {, ¿qué puede significar? Proponga } \\
\text { una definición y un modo de determinarlo. }\end{array}$ \\
\hline
\end{tabular}


añadidos, lo que centraría la atención del alumno en el análisis de dicha gráfica.

6) Continuando con el proceso de resolución podemos proponer a los alumnos el análisis de la resolución que se plantea desde una metodología que reduzca los equilibrios según interese para resolver el problema matemático, es decir, analizar la resolución que proponen algunos libros en los que se insiste en obtener el resultado numérico mediante aproximaciones sucesivas. Con esta actividad se pretende que el alumno compare las dos opciones a la hora de resolver estos problemas y, también, insistir en las consecuencias que puede tener en su aprendizaje si se conforma con las aproximaciones, ya que supone un alejamiento de la realidad que sucede: al eliminar equilibrios químicos porque no interesan para el proceso de resolución, se concede más importancia a unos que a otros y con el paso del tiempo terminamos por no considerarlos.

7) Hasta ahora nos hemos centrado en el proceso de resolución; sin embargo, un aspecto importante que todavía no hemos introducido es que el docente tiene que proponer problemas de temática diversa, de forma que no se plantee el cálculo de una incógnita $\left(\mathrm{pH}\right.$ o $\left.\left[\mathrm{H}_{3} \mathrm{O}^{+}\right]\right)$, sino solicitar que se determine la concentración de todas las especies.

En la tabla V, mostramos algunos problemas que pueden resolverse siguiendo la secuencia propuesta anteriormente. También se exponen los objetivos que surgen de dar respuesta a las dificultades de aprendizaje detectadas en la fase de diagnóstico del presente artículo.

8) Hasta el momento hemos presentado pocas actividades relacionadas con los indicadores ácido-base. Dada esta escasez, queremos plantear dos prácticas diseñadas como problemas experimentales que tengan como objetivos favorecer la comprensión de algunos contenidos relacionados con los indicadores en los que hemos detectado algunas dificultades, tales como no considerar el equilibrio del indicador, presente en todo el proceso, ni el intervalo de viraje, donde el color que se aprecia es la mezcla de los colores de las dos formas del indicador.

Los objetivos a cubrir por estas prácticas pueden ser muy diversos como, por ejemplo, la adquisición de habilidades manipulativas, que se centran en los contenidos procedimentales; la vivencia de fenómenos que favorecen la comprensión de contenidos conceptuales; así como el incremento de la motivación y de una actitud positiva hacia la ciencia.

Sin embargo, las actividades que se proponen en el laboratorio no siempre desarrollan estos objetivos sino que, como algunos autores señalan (Hodson, 1994; Yager y Penick, 1987), el diseño tradicional de experiencias corresponde a tareas rutinarias, en las que los alumnos no realizan actividades creativas sino que, más bien, únicamente siguen las indicaciones de una receta.

Por ello, el trabajo práctico debe estar muy bien diseñado para conseguir no sólo que el alumno se acerque y conozca una parte fundamental en el trabajo de los científicos, como es el trabajo en el laboratorio, sino que también adop- te una actitud positiva hacia la ciencia y desarrolle habilidades tanto de tipo manual (operativas) como intelectual (resolución de situaciones problemáticas), ya que la gran inversión de tiempo, energías y recursos que se necesitan para su montaje deben ser justificados.

$\mathrm{Al}$ proyectar las prácticas como problemas se puede emplear en el laboratorio el mismo método heurístico utilizado para la resolución de problemas cuantitativos y cualitativos que hemos comentado ampliamente en párrafos anteriores. La única diferencia es que en la etapa de ejecución se requiere el diseño de un montaje experimental y, por tanto, el manejo de habilidades procedimentales. A modo de ejemplo, algunos de los problemas sugeridos en la tabla $\mathrm{V}$ pueden y deben ser resueltos utilizando el laboratorio como medio.

A continuación mostraremos diferentes formas de plantear una práctica de laboratorio sobre los indicadores. Los docentes deben elegir la forma (cerrada, semiabierta o abierta) que prefieran para lograr los objetivos que se propongan con la actividad.

Con la siguiente práctica no se observa un cambio brusco del indicador, sino que cambian los colores gradualmente, por lo que se facilita el aprendizaje del concepto intervalo de viraje.

En primer lugar, describimos la práctica de una forma tradicional (Rendle, Vokins y Davis, 1972):

\section{Intervalo de $\mathrm{pH}$ de los indicadores}

Se necesita material para valoraciones, disolución tampón universal, disolución de hidróxido de sodio $(0,1 \mathrm{M})$ e indicadores (metilnaranja, timolftaleina, fenolftaleína, etc.). Método: Prepare el color ácido mezclando $50 \mathrm{~cm}^{3}$ de agua pura en un erlenmeyer con $2,5 \mathrm{~cm}^{3}$ de ácido clorhídrico $2 \mathrm{M}$ y añadiendo exactamente 5 gotas de un indicador. Prepare el color básico de la misma forma pero usando hidróxido de sodio $2 \mathrm{M}$. Diluya a $25 \mathrm{~cm}^{3}$ una alícuota de la disolución reguladora con $10 \mathrm{~cm}^{3}$ de agua pura y añada exactamente 5 gotas de un indicador. Añada a la mezcla del tampón el hidróxido de sodio $(0,1 \mathrm{M})$ hasta que el primer indicador comience a cambiar desde su color ácido. Anote la lectura de la bureta. Con el objeto de realizar una buena comparación, las disoluciones coloreadas deben ser diluidas con agua pura lo necesario para tener aproximadamente el mismo volumen que la mezcla a valorar. Utilice la siguiente relación para calcular el intervalo de $\mathrm{pH}$ en el que el indicador cambia de color: $\mathrm{pH}=3,1+0,237 \mathrm{~V}$, donde $\mathrm{V}=\mathrm{cm}^{3}$ de $\mathrm{NaOH}(\mathrm{ac}) 0,1 \mathrm{M}$ añadido hasta un máximo de $35 \mathrm{~cm}^{3}$. Esta relación ha sido tomada de la valoración de una alícuota de $25 \mathrm{~cm}^{3}$ de la disolución reguladora universal utilizando como valorante hidróxido sódico 0,1M. Sin embargo, no es preciso conocer una ecuación determinada sino que se puede utilizar como alternativa el pehachímetro (o incluso que los alumnos calculen la relación entre el volumen y el $\mathrm{pH}$ según distintas disoluciones reguladoras).

Esta tarea puede ser transformada en un problema experimental semiabierto con el siguiente enunciado:

«Utilice una disolución reguladora y varios indicadores para poner de manifiesto que el color que se aprecia en el intervalo de viraje es una mezcla de los colores de las dos formas del indicador.»

Por último, podemos plantear el problema abierto con el siguiente enunciado: 
«Todos los indicadores poseen un intervalo de viraje donde el color que se observa es la mezcla de los colores de las dos formas del indicador. Diseñe un experimento que ponga de manifiesto esta afirmación.»

Para terminar esta propuesta de actividades queremos insistir en la necesidad de que los alumnos indaguen de forma experimental que el equilibrio entre las especies del indicador ocurre durante todo el proceso. Para ello, se pueden utilizar medidas espectrofotométricas, de forma que se pueda observar que, para valores fuera del intervalo de viraje de un indicador están presentes las dos formas.

Al igual que en la práctica anterior, se pueden plantear tres enunciados diferentes según se quiera un experimento cerrado, semiabierto o abierto:

- Cerrado: Tome los siguientes indicadores: fenolftaleína $\mathrm{y}$ verde de bromocresol. Mida utilizando un espectrofotómetro a dos unidades del comienzo del intervalo de viraje, en el centro del intervalo de viraje y dos unidades posteriores a dicho intervalo.

- Semiabierto: Medidas espectrofotométricas de varios indicadores a varios valores del $\mathrm{pH}$.

- Abierto: Diseñe una experiencia para reconocer las dos formas del indicador durante todo el proceso.

\section{IMPLICACIONES PARA LA ENSEÑANZA UNIVERSITARIA}

El diagnóstico de las concepciones alternativas pone de manifiesto algunas deficiencias de la enseñanza universitaria actual que, como destacan Perales (1998), Gil y Vilches (1999) o Campanario (2002), comienza con la formación didáctica inicial y permanente del profesorado universitario de ciencias. Una de las ideas que más se destaca en la investigación es que, en la formación inicial del profesorado, se debe incidir en el conocimiento didáctico del contenido, dotando al profesorado de una carga profesional elevada (como profesores de ciencias y no como profesionales en ciencias), de forma que los contenidos estén estrechamente ligados a la didáctica de las ciencias experimentales sin que esta formación del profesorado sea sumativa (contenidos de ciencias con una coletilla final de aplicación didáctica) sino que debe ser de forma globalizada, no fragmentada (Mellado, 2000).

Como nuestra tarea investigadora no sólo es detectar problemas sino también realizar propuestas de mejora, las concepciones alternativas descritas permiten fundamentar propuestas de enseñanza que faciliten el aprendizaje de los contenidos implicados. La generación de conflictos sobre contenidos concretos al nivel universitario evita caer en una formación didáctica general de la que huyen los docentes universitarios de ciencias, creando conexiones a través de la utilidad para sus clases, al mismo tiempo que permite la reflexión sobre su conocimiento didáctico declarado y su práctica docente.

Con respecto a los equilibrios ácido-base múltiples, las propuestas deben tratar de salvar las principales dificultades de los estudiantes, incluidos los universitarios, quienes no consideran la simultaneidad de todos los procesos que transcurren y conceden más importancia a unos equilibrios y eliminan otros porque no son necesarios para obtener un resultado numérico a la hora de resolver los cálculos que se suelen pedir en los problemas de equilibrios múltiples.

El planteamiento y la resolución mediante excesivas aproximaciones de algunos problemas, por tanto, puede inducir al razonamiento causal secuencial, no considerar todos los equilibrios, el escaso control de variables o, por el contrario, puede convertirse en una excelente ocasión para facilitar el aprendizaje de los contenidos relacionados con los equilibrios múltiples, entre ellos la simultaneidad que los caracteriza, que todos transcurren desde el principio del proceso, la presencia de todas las especies posibles en los diferentes equilibrios, etc. Por ello, nuestra propuesta se centra en plantear problemas de temática diversa y ofrecer un método heurístico para su resolución con el objetivo de que los estudiantes utilicen todas las variables puestas en juego en estos procesos y no reduzcan los problemas a la obtención de un resultado numérico que se obtiene por múltiples aproximaciones matemáticas.

Por todo ello, el planteamiento y las estrategias de resolución de problemas que proponemos es una llamada de atención al estudiante, $-\mathrm{y}$ al profesorado universitario de ciencias químicas- sobre la importancia de evaluar de forma cualitativa el problema químico (las especies, los equilibrios, las concentraciones de las especies, las relaciones que se establecen, etc.) y reducir los aspectos matemáticos con ayuda del ordenador.

Esta investigación no sólo plantea una mejora en el aprendizaje de los equilibrios múltiples al nivel universitario, sino que también permite aprovechar la ocasión para hacer un llamamiento a favor de la mejora de la formación del profesorado (también del universitario). De este modo, las diferencias entre los contenidos sobre ácidos y bases en los niveles secundario y universitario deben ser marcadas para evitar las consecuencias nefastas (porque perduran) de adelantar contenidos que, por su dificultad, deben reservarse a niveles educativos superiores: el hecho de introducir los equilibrios múltiples en bachillerato (justo después de la introducción de los contenidos relacionados con los equilibrios «simples») conlleva la utilización del razonamiento causal descrito para resolver los problemas y poder obtener un resultado numérico satisfactorio y una explicación química convincente. 


\section{NOTA}

${ }^{1}$ En párrafos anteriores citamos algunos ejemplos de las investigaciones neopiagetianas de Niaz sobre la demanda-M de los problemas de química. Este autor se centra en los problemas estequiométricos y de equilibrios. Asumimos que, si los problemas de equilibrios poseen una elevada demanda-M, ésta se verá aumentada al tratar con equilibrios múltiples porque aumentan las variables (el número de equilibrios, el número de especies que es preciso controlar de forma simultánea). El problema de los equilibrios múltiples tiende a resolverse utilizando el razonamiento causal secuencial antes descrito porque resulta una estrategia de enseñanza y de aprendizaje mucho más económica (de menor demanda).

\section{REFERENCIAS BIBLIOGRÁFICAS}

ANAMUAH-MENSAH, J. (1986). Cognitive strategies used by chemistry students to solve volumetric analysis problems. Journal of Research in Science Teaching, 23(9), pp. 759-769.

ANDERSSON, B. (1990). Pupils' conceptions of matter and its transformations (age 12-16). Studies in Science Education, 18, pp. 54-85.

BABER, J. (1996). Of cabbages and Chemistry. Lawrence Hall of Science. Berkeley.

BLONDEL, F.M. y SCHWOB, M. (1996). Expertise pédagogique et modélisation de l'élève dans un environnement de résolution de problèmes de chimie. Tecnologies nouvelles et éducation. Documents et travaux de recherche en éducation. INRP.

BOUJAODE, S. (1993). «Students' systematic errors when solving kinetic and chemical equilibrium problems.» Comunicación presentada en el Annual Meeting of the National Association for Research in Science Teaching. Atlanta.

CAMPANARIO. J.M. (2002). Asalto al castillo: ¿A qué esperamos para abordar en serio la formación didáctica de los profesores universitarios de ciencias? Enseñanza de las Ciencias, 20(2), pp. 315-325.

COBB, V. (1998). Cobb's red cabbage indicator. Chemecology, 27(2), pp. 15.

CROS, D. et al. (1986). Conceptions of first year university students of the constitution of matter and the notions of acids and bases. European Journal of Science Education, 8(3), pp. 305-313.

CROS, D. et al. (1988). Conceptions of second year university students of some fundamental notions in Chemistry. International Journal of Science Education, 10(3), pp. 331336.

DE LA GUARDIA, M., SALVADOR, A., LÓPEZ, J. y CARRION, J.L. (1985). Errores conceptuales en la concepción de los equilibrios ácido-base. Enseñanza de las Ciencias, núm. extra, p. 61 .
DE MANUEL, E., JIMÉNEZ-LISO, M.R. y SALINAS, F. (2000). Las concepciones sobre ácidos y bases de los opositores al cuerpo de profesores de secundaria. Alambique, 24, pp. 6676.

DRIVER, R., GUESNE, E. y TIBERGHIEN, A. (1989). Ideas científicas en la infancia y la adolescencia. Madrid: MECMorata.

FERNÁNDEZ GONZÁLEZ, M. (2000). Problemas de química, en Perales, F.J. La resolución de problemas en ciencias experimentales, pp. 140-169. Madrid: Síntesis.

FORD, N. (2000). Cognitive styles and virtual environments. Journal of the American Society for Information Science, 51(6), pp. 543-557.

FORTMAN, J.J. (1994). Pictorial analogies XI: concentrations and acidity of solutions. Journal of Chemical Education, 71(5), pp. $430-432$.

GIL, D. y VILCHES, A. (1999). Problemas de la educación científica en la enseñanza secundaria y la universidad: contra las evidencias. Revista Española de Física, 13(5), pp. 10-15.

HOBDEN, P. (1998). The role of routine problem tasks in Science Teaching, en Fraser, B.J. y Tobin, K.G. International Handbook of Science Education, pp. 219-232. Dordercht: Kluwer A.P.

HODSON, D. (1994). Hacia un enfoque más crítico del trabajo de laboratorio. Enseñanza de las Ciencias, 12(3), pp. 299-313.

HUDDLE, P.A. y PILLAY, A.E. (1996). An in-depth study of misconceptions in stoichiometry and chemical equilibrium at South African University. Journal of Research in Science Teaching, 33(1), pp. 65-77.

JIMÉNEZ-LISO, M.R. (2000). «Contenidos relacionados con los procesos ácido-base: diagnóstico y propuestas didácticas al nivel universitario». Tesis doctoral no publicada. Facultad de Ciencias de la Educación. Universidad de Granada. 
JIMÉNEZ-LISO, M.R., DE MANUEL, E., GONZÁLEZ, F. y SALINAS, F. (2000). La utilización del concepto del pH en la publicidad y su relación con las ideas que manejan los alumnos: aplicaciones en el aula. Enseñanza de las Ciencias, 18(3), pp. 451-461.

JIMÉNEZ-LISO, M.R., DE MANUEL, E. y SALINAS, F. (2001). Las concepciones sobre los ácidos y las bases: su tratamiento en los textos de los niveles universitario y medio, en Martín, M. y Morcillo, J.G. (eds.). (2001). Reflexiones sobre la Didáctica de las Ciencias Experimentales. Actas de los XIX Encuentros de Didáctica de las Ciencias Experimentales, pp. 454-462. Madrid: Nivola.

JIMÉNEZ-LISO, M.R. y DE MANUEL, E. (2002). La neutralización ácido-base a debate. Enseñanza de las Ciencias, 20(3), pp. 451-464.

JONASSEN, D.H. (1996). Computers in the classroom. Nueva Jersey: Prentice-Hall.

KANDA, N. et al. (1995). Preparing «Chamaleon Balls» form natural plants: simple hadmade $\mathrm{pH}$ indicator and teaching material for chemical equilibrium. Journal of Chemical Education, 72(12), pp. 1131-1132.

KEMPA, R.F. (1986). Resolución de problemas de química y estructura cognitiva. Enseñanza de las Ciencias, 4(2), pp. 99110 .

KUMAR, D.D. (1993). Assessment of Expert-Novice Chemistry problem solving using Hypercard: early findings. Journal of Science Education and Technology, 2(3), pp. 481-485.

KUMAR, D.D. et al. (1994). A study of the effect of Hypercard and pen-paper performance assessment methods on expertnovice Chemistry problem solving. Journal of Science Education and Technology, 3(3), pp. 187-200.

MASON, D.S. y CRAWLEY, F.E. (1994). «Differences between algorithmic and conceptual problem solving by nonscience majors in Introductory Chemistry.»Comunicación presentada en Annual Meeting of the National Association for Research in Science Teaching. Anaheim.

MASON, D.S., SHELL, D.F. y CRAWLEY, F.E. (1997). Differences in problem solving by nonscience majors in Introductory Chemistry on paired algorithmic conceptual problems. Journal of Research in Science Teaching, 34(9), pp. $905-924$.

MELLADO, V. y GONZÁLEZ, T. (2000). La formación inicial del profesorado de ciencias, en Perales y Cañal (eds.). Didáctica de las Ciencias Experimentales, pp. 535-555. Alcoy: Marfil.

NAKHLEH, M.B. y KRAJCIK, J.S. (1994). Influence of levels of information as presented by different technologies on students' understandings of acid, base and $\mathrm{pH}$ concepts. Journal of Research in Science Teaching, 31(10), pp. 10771096.

NIAZ, M. (1988). The information processing demand of Chemistry problems and its relation to Pascual-Leone's functional M-capacity. International Journal of Science Education, 10(2), pp. 231-238.

NIAZ, M. (1989). Dimensional Analysis: A Neo-Piagetian Evaluation of M-demand of Chemistry Problems. Research in Science and Technological Education, 7(2), pp.153-170. (ERIC Identifier: EJ403037)
NIAZ, M. (1993). «Reasoning strategies of students in solving Chemistry problems as a function of developmental level, functional M-capacity and disembedding ability.» Comunicación presentada en Annual Meeting of the National Association for Research in Science Teaching (Atlanta, GA, April, 1993). (ERIC Identifier: ED360180).

PASCUAL-LEONE, J. (1978). Compounds, confounds and models in developemental information processing: a reply to Trabasso and Foellinger. Journal of Experimental Child Psychology, 26, pp. 18-40.

PASCUAL-LEONE, J. (1987). Organismic processes for NeoPiagetian theories: a dialectical causal account of cognitive development. International Journal of Psichology, 22, pp. 531570 .

PASK, G. (1976). Styles and strategies of learning. British Journal of Educational Psychology, 46, pp. 128-148.

PASK, G. (1988). Learning strategies, teaching strategies, and conceptual or learning style, en Schmeck, R.R. (ed.). Learning strategies and learning styles, pp. 8-99. Nueva York: Plenum Press.

PASK, G., y SCOTT, B.C.E. (1972). Learning strategies and individual competence. International Journal of Man-Machine Studies, 4, pp. 217-253.

PASK, G. y SCOTT, B.C.E. (1973). CASTE: A system for exhibiting learning strategies and regulating uncertainty. International Journal of Man-Machine Studies, 5, pp. 17-52.

PERALES, F.J. (1994). Enseñanza-aprendizaje de una heurística en la resolución de problemas en física: un estudio cuasi experimental. Revista Interuniversitaria de Formación del Profesorado, 21, pp. 201-209.

PERALES, F.J. (1998). La formación del profesorado universitario en didáctica de las ciencias experimentales. Desde el inmovilismo a la búsqueda de alternativas. Revista de Educación de la Universidad de Granada, 11, pp. 345-354.

PERALES, F.J. (2000). La resolución de problemas en ciencias experimentales. Madrid: Síntesis.

POLYA, G. (1945). How to solve it. Princeton University Press. Nueva Jersey. Citado en Perales, F.J. (2000), p. 37.

RENDLE, P., VOKINS, M. y DAVIS, P. (1972). Experimental Chemistry. Londres: Arnold.

ROSS, B. y MUNBY, H. (1991). Concept mapping and misconceptions: a study of high-school students' understandings of acids and bases. International Journal of Science Education, 13(1), pp. 11-23.

SANMARTÍ, N. (1996). Para aprender ciencias hace falta aprender a hablar sobre las experiencias y sobre las ideas. Textos, 8, pp. 27-39.

SCHMIDT, H.J. (1991). A label as a hidden persuader: chemists' neutralization concept. International Journal of Science Education, 13(4), pp. 459-471.

SHERWOOD, R.D., PETROSINO, A.J., LIN, X. et al. (1998). Problem-based macro contexts in Science Instruction: Design issues and applications, en Fraser, B.J. y Tobin, K.G. International Handbook of Science Education, pp. 349-362. Kluwer A.P. Dordercht. 
SIERRA, J.L. (2000). Informática y enseñanza de las ciencias, en Perales, F.J. y Cañal, P. La Didáctica de las ciencias experimentales: teoría y práctica de la enseñanza de las ciencias, pp. 339-359. Alcoy: Marfil.

TOPLIS, R. (1998). Ideas about acids and alkalis. School Science Review, 80(291), pp. 67-70.

UZELMEIER, C.E. y BREYER, A.C. (1998). Red shoe-blue shoe: an acid-base demonstration with a fashionable twist. Journal of Chemical Education, 72(2), pp. 183-184.

VANCLEAVE, J. (1998). Hands-on science: Is it an acid or a base? These colorful tests tell all! Instructor (Primary), 107(6), pp. 97-98.

VIDAL DE LABRA, J.A., ROMERO, F. y REQUENA, A. (1985). Enseñanza basada en ordenador: una experiencia en BUP. Enseñanza de las Ciencias, 2, pp. 100-107.
VIDYAPATI, T.J. y SEETHRAMAPPA, J. (1995). Higher secondary school student's concepts of acids and bases. School Science Review, 77(278), pp. 82-84.

VIENNOT, L. (1979). Spontaneous Reasoning in Elementary Dynamics. European Journal of Science Education, 2, pp. 205-214.

YAGER, R.E. y PENICK, J.E. (1987). Resolving the Crisis in Science Education: Understanding before Resolution. Science Education, 71(1), pp. 49-55.

ZABALZA, M. (1985). Teoría y práctica del diseño curricular. Universidad de Santiago de Compostela.

ZOLLER, U. (1990). Students' misunderstandings and misconceptions in college freshman chemistry (General and Organic). Journal of Research in Science Teaching, 27(10), pp. 1053-1065.

[Artículo recibido en noviembre de 2001 y aceptado en enero de 2003.] 